\title{
La génération de solides divisés par fluides supercritiques
}

\author{
Jacques Fages*, Hubert Lochard, Elisabeth Rodier, Jean-Jacques Letourneau et \\ Martial Sauceau
}

Ecole des Mines d'Albi-Carmaux, Laboratoire de Génie des Procédés des Solides Divisés- UMR CNRS 239281013

ALBI, FRANCE

\section{Génération de solides divisés : limites des procédés traditionnels et avantages des procédés utilisant les FSC}

Les solides divisés sont très largement utilisés dans le monde industriel. Quel que soit le secteur : industrie minérale, chimie fine, cosmétiques, industries agroalimentaires, peintures, industrie pharmaceutique, détergents, ... les poudres y sont omniprésentes.

Il existe deux grandes voies d'obtention de fines particules :

- par réduction de taille : broyage, comminution, micronisation ;

- par cristallisation : précipitation, atomisation, cristallisation par évaporation, refroidissement ou variation de force ionique.

\section{Réduction de taille}

En raison de leur relative simplicité, les procédés de broyage sont toujours extrêmement utilisés dans l'industrie. Cependant, ils ne sont pas dépourvus d'inconvénients qui peuvent s'avérer gênants voire rédhibitoires :

- leur rendement énergétique est très faible ;

- il existe un risque de pollution par l'outil de broyage ;

- le contrôle de la taille des particules est limité (génération de fines non désirées) ;

- il n'y a pas de contrôle de la morphologie particulaire ;

- la distribution de taille des particules est large ;

- le procédé n'est pas utilisable dans le cas de produits thermosensibles ou explosifs ;

- il existe une taille limite inférieure, de l'ordre de quelques micromètres, en deçà de laquelle il est extrêmement difficile de descendre ;

- dans certains cas particuliers il faut utiliser un adjuvant de broyage ;

- au moins une étape aval de tamisage est nécessaire.

\section{Cristallisation en milieu liquide}

La seconde voie principale permet la génération du solide à partir d'une phase fluide. Plusieurs procédés empruntent cette voie, les plus emblématiques étant :

- I'atomisation : le liquide (l'eau le plus souvent) est évaporé par un très court passage à température élevée, conduisant à un résidu solide
Les procédés de cristallisation utilisant les fluides supercritiques (FSC) constituent une nouvelle voie pour obtenir des solides finement divisés. En utilisant principalement la pression comme paramètre opératoire, ces procédés permettent l'obtention de poudres fines et monodisperses. On distingue deux familles de procédés selon que le fluide supercritique généralement le $\mathrm{CO}_{2}-$ est utilisé comme solvant (RESS) ou comme anti-solvant (SAS). Pour le procédé RESS, la chute de densité liée à une détente brutale est le moteur de la nucléation. Pour les procédés de type SAS c'est la dissolution réciproque d'un solvant organique et du FSC qui provoque la précipitation. Des applications industrielles se dessinent dans de nombreux secteurs, même si c'est l'industrie pharmaceutique qui semble la mieux placée pour rassembler une majorité de ses développements.

Processes using crystallisation from a supercritical medium constitute a new route to obtain finely divided solids. By using the pressure as an operating parameter, these processes allow to obtain fine and monodisperse powders. There exist two types of processes, according to whether supercritical fluid usually carbon dioxide - is used as a solvent or an anti-solvent: RESS or SAS. In the first case it is the drop in density due to the brutal decompression of the fluid which is the driving force of nucleation, in the second it is the reciprocal dissolution of an organic solvent in the supercritical fluid. Industrial applications are expected in many sectors, even if it is the pharmaceutical industry which seems best armed to gather a majority of its developments.

Mots clés : fluides supercritiques, cristallisation, poudres, solides divisés, RESS, SAS.

\footnotetext{
* Toute correspondance doit être adressée à cet auteur. Courriel jacques.fages@enstimac.fr
} 
composé des substances préalablement dissoutes dans le milieu ;

- la précipitation : une réaction chimique mettant en jeu deux réactifs (ou plus) conduit à la formation d'une phase solide insoluble dans le liquide ;

- la cristallisation : en modifiant la température, le $\mathrm{pH}$, la force ionique ou encore par évaporation du solvant, des nuclei de particules solides se forment et peuvent croître pour finalement donner un solide humide.

Cette famille de procédés présente également des inconvénients :

- pas ou peu de contrôle du polymorphisme cristallin ;

- présence de solvant résiduel ;

- nécessité d'une étape complémentaire de séchage.

Enfin, il faut noter que tous ces procédés sont opérés à pression atmosphérique, celle-ci n'étant donc pas une variable. Il s'agit là d'une des différences majeures avec les procédés utilisant les FSC.

\section{Procédés utilisant les FSC}

La technologie des fluides supercritiques constitue une nouvelle et troisième voie d'obtention de solides sous forme divisée (Adschiri, 1998). Bien que la possibilité d'obtenir une poudre à partir d'un fluide comprimé soit connue depuis plus de 120 ans (Hannay et Hogarth, 1879), c'est seulement depuis le milieu des années 1980 que des articles scientifiques ont été publiés en vue de comprendre et d'utiliser ces propriétés. Une des particularités les plus remarquables de ces procédés est l'utilisation de la pression comme paramètre opératoire. La plupart des inconvénients précédemment signalés pour les procédés traditionnels peuvent être évités. De plus, les procédés utilisant des FSC permettent I'obtention sous forme de poudres fines de produits impossibles à microniser autrement.

Ainsi, des micro- voire des nano-particules avec une distribution de taille resserrée sont obtenues par l'utilisation de ce type de procédé qui est également bien adapté à la micro-encapsulation ou au revêtement de surface de particules par un polymère.

Ces procédés sont généralement classés en deux familles, selon que le fluide supercritique est utilisé comme solvant (procédés RESS et dérivés) ou comme anti-solvant (procédés SAS et dérivés).

\section{Le procédé RESS}

\section{Principe du procédé}

RESS est I'acronyme signifiant "Rapid Expansion of Supercritical Solutions". Ce procédé est parfois qualifié d'atomisation supercritique (Subra et Jestin, 1999). Son principe est d'une grande simplicité : il utilise les propriétés de solvatation d'un FSC pour un solide donné. Le pouvoir solvant d'un fluide dépend de sa densité (Van Alsten et Eckert, 1993), que I'on peut faire varier dans de grandes proportions en jouant sur la température et la pression. Dans la région supercritique un fluide est à la fois dense et compressible, et peut, selon les conditions opératoires, se révéler un bon ou un piètre solvant. En conséquence, un solide peut être cristallisé à partir d'une solution homogène dans un FSC par simple détente. Les conditions opératoires de pression et de température sont choisies pour que le mélange solvant-soluté se présente, avant la détente, sous la forme d'une seule phase homogène. Cette détente est effectuée par passage à travers un orifice, qui peut être un simple trou circulaire, un capillaire ou encore une buse. En général, on choisit un orifice de très faible diamètre, souvent

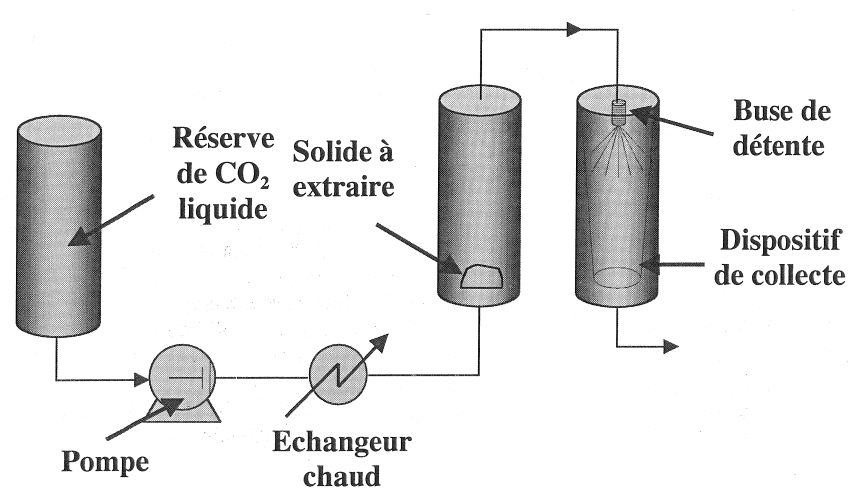

Figure 1. Schéma de principe du procédé RESS

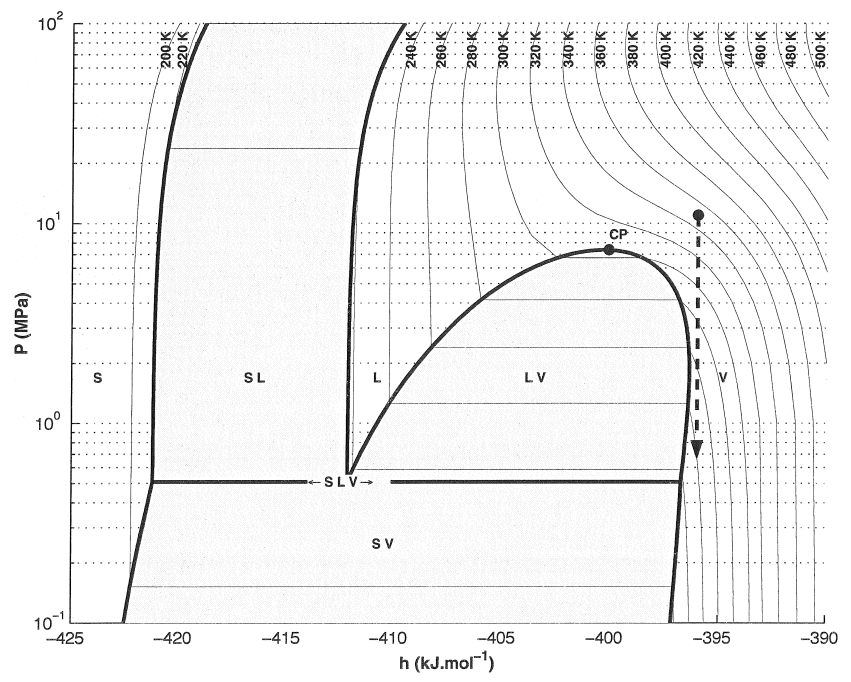

Figure 2. Détente lors du procédé RESS dans le diagramme enthalpiepression pour le $\mathrm{CO}_{2}$ pur.

de quelques dizaines à quelques centaines de $\mu \mathrm{m}$, afin de provoquer une forte chute de la pression. À moins d'apporter de l'énergie, la détente est quasi-adiabatique et provoque une forte chute de la densité. Le fluide se retrouve alors à l'état gazeux avec un très faible pouvoir solvant. Le schéma de principe du procédé est donné sur la Figure 1.

\section{Optimisation du procédé RESS Étape d'extraction}

Celle-ci passe obligatoirement par une bonne connaissance de la première étape du procédé : la dissolution du solide dans un FSC. Pour une revue sur ce sujet, on pourra se reporter à Mc Hugh et Krukonis (1994) ou Lucien et Foster (2000). Il est donc indispensable de collecter des données sur les équilibres entre phases avec les composés en présence. Ces données existent parfois dans la littérature, mais il faut souvent procéder à des déterminations expérimentales. Ces données permettent d'ajuster les paramètres des modèles de comportement de ces solutions dont un résultat essentiel à l'optimisation de cette étape d'extraction est la connaissance de la solubilité du solide étudié dans le fluide considéré en fonction des conditions 
opératoires (Sauceau et al., 2000a). Le solvant peut être un fluide pur (généralement le $\mathrm{CO}_{2}$ ) ou un mélange lorsqu'un co-solvant est ajouté.

Dans le cas simple d'un mélange binaire solide-FSC, une expression possible de la solubilité du solide dans le fluide, exprimée en fraction molaire, est donnée par l'équation suivante où l'indice 2 fait référence au soluté :

$$
y_{2}=\frac{P_{2}^{s a t}}{\varphi_{2} P} \exp \left[\frac{V_{2}^{s}}{R T}\left(P-P_{2}^{s a t}\right)\right]
$$

Le volume molaire du solide pur, $V_{2}{ }^{s}$, ne dépend pas de la pression $P$ et peut être assez facilement mesuré. Le coefficient de fugacité du soluté dans la phase fluide, $\varphi_{2}$, exprime la déviation à l'idéalité de la fugacité notée $f$. $\varphi_{2}$ peut être calculé à l'aide d'une équation d'état. La fugacité du soluté dans un fluide considéré comme un gaz parfait s'exprime sous la forme $: f_{2}=P y_{2}$, tandis que dans un fluide réel cette expression devient $: f_{2}=\varphi_{2} P y_{2}$.

La pression de sublimation du soluté $P_{2}$ sat , qui est fonction de la température, est en général inconnue mais peut elle aussi être calculée à l'aide d'une équation d'état (Neau et al., 1999).

Une autre approche est souvent utilisée pour des molécules dont les données thermodynamiques sont inconnues. II s'agit de l'utilisation de modèles empiriques basés sur des corrélations entre la solubilité et la densité (Chrastil, 1982 ; Méndez-Santiago et Teja, 2000).

Les variables d'état qui gouvernent l'étape d'extraction, et sur lesquelles il est possible d'agir directement, sont bien sûr la température et la pression. Le débit du fluide est également important car il peut conditionner l'atteinte d'un équilibre thermodynamique dans l'autoclave d'extraction. En effet, on ne peut négliger la cinétique de dissolution dans le cas où des limitations diffusionnelles sont rencontrées. Enfin, le composé à extraire peut ne pas être un corps pur, un cas typique étant celui d'une graisse ou d'un polymère. Dans ce cas, une extraction sélective et un fractionnement du solide peuvent avoir lieu, induisant une variation dans la composition des particules formées lors de l'étape de détente.

\section{Étape de détente}

L'utilisation d'un diagramme enthalpie-pression, connu sous le nom de diagramme de Mollier, peut s'avérer d'une grande utilité quant à la compréhension du phénomène de détente. La Figure 2 présente le diagramme de Mollier du $\mathrm{CO}_{2}$ pur. Si la variation d'énergie cinétique dans la buse de détente est négligée, la détente peut être considérée comme isenthalpe. Sur la Figure 2, la chute de pression sera donc représentée par une ligne verticale. On constate qu'elle s'accompagne d'une forte chute en température. Dans l'exemple donné, les points de départ et d'arrivée se trouvent dans la zone monophasique, il convient en effet d'éviter toute apparition de phase liquide susceptible de re-solubiliser la poudre produite. Cette exigence suppose une température de pré-expansion élevée, ce qui peut s'avérer difficile à mettre en œuvre avec des solutés thermosensibles. Abaisser cette température (c'est-à-dire se déplacer vers la gauche dans le diagramme) peut conduire à la liquéfaction du fluide ou même à la formation de glace carbonique (la pression du point triple du $\mathrm{CO}_{2}$ est à $0,52 \mathrm{MPa}$ ), avec les risques de colmatage que cela comporte. Pour résoudre ce problème, il est possible soit d'utiliser une buse chauffante, soit encore de pré-chauffer le fluide juste avant son entrée dans la buse. Dans ce cas, il faut pouvoir calculer ou mesurer la quantité de chaleur effectivement transmise au fluide si I'on veut savoir où l'on se situe sur un diagramme enthalpique. Une autre possibilité est de choisir des couples $(T, P)$ avant et après détente permettant d'éviter les problèmes précités.

La nucléation du soluté intervient lors de la brutale détente, qui s'accompagne d'une onde de choc. C'est cette perturbation mécanique se propageant à la vitesse du son qui est responsable des conditions uniformes expliquant la faible dispersion dans les tailles de particules obtenues (Tom et Debenedetti, 1991). En fait, le temps d'expansion ne dépend pas seulement de la vitesse du son (qui varie avec la nature du fluide et les conditions opératoires) mais aussi de la longueur de l'orifice de détente. Avec une buse capillaire, Smith et al. (1986) ont proposé une approximation entre $10^{-4}$ et $10^{-6}$ seconde. C'est durant ce très bref instant que la solubilité chute de plusieurs ordres de grandeur provoquant des niveaux très élevés de sursaturation. La sursaturation est le terme traduisant une différence de potentiel chimique $\mu_{i}$ du corps $i$ considéré entre la solution et les cristaux, ce qui induit un transfert de matière de I'une vers les autres. Cette différence de potentiel est exprimée dans l'équation (2).

$$
\begin{aligned}
& \Delta \mu_{i}=R T \ln \frac{\gamma_{i} c_{i}}{\gamma_{i}^{*} c_{i}^{*}} \\
& \Delta C i=c i-c_{i}^{*} \\
& \sigma_{i}=\frac{c_{i}-c_{i}^{*}}{c_{i}^{*}}=S-1 \\
& S=\frac{c_{i}}{c_{i}^{*}}
\end{aligned}
$$

Comme l'indique l'équation (2), il faut tenir compte des coefficients d'activité $\gamma$. Cependant, dans le cas fréquent où la solubilité du corps considéré est faible, ces coefficients sont proches de 1 et peuvent être négligés. La sursaturation absolue $\Delta c$ et la sursaturation relative $\sigma$ sont alors définies selon les équations (3) et (4), en utilisant la concentration du soluté $c_{i}$ et sa concentration à saturation ( $c^{\prime}$ est-à-dire sa solubilité) $C^{*}$. On utilise aussi souvent le rapport de sursaturation noté $S$ (Eq. 5)

La sursaturation relative ou absolue est donc le paramètre représentant la force motrice de cristallisation (Klein et David, 1995). À proximité du point critique, la variation de la densité due à une baisse de pression peut s'avérer considérable et explique les très importantes valeurs de sursaturation obtenues. Le fluide après dépressurisation pouvant être assimilé à un gaz parfait, la variation de solubilité du soluté dans le fluide peut être quantifiée à l'aide du facteur d'augmentation $E$ ainsi défini :

$$
E=\frac{P y_{2}}{P_{2}^{s a t}}
$$

$y_{2}$ est la fraction molaire du soluté dans le fluide, $P$ la pression en amont de la buse et $P_{2}$ sat la pression de sublimation, c'est-à-dire la tension de vapeur, du solide dans un gaz parfait. Le facteur $\mathrm{d}^{\prime}$ augmentation peut prendre des valeurs de l'ordre de $10^{5}$ à $10^{6}$ donnant une idée des niveaux de sursaturation atteints. 


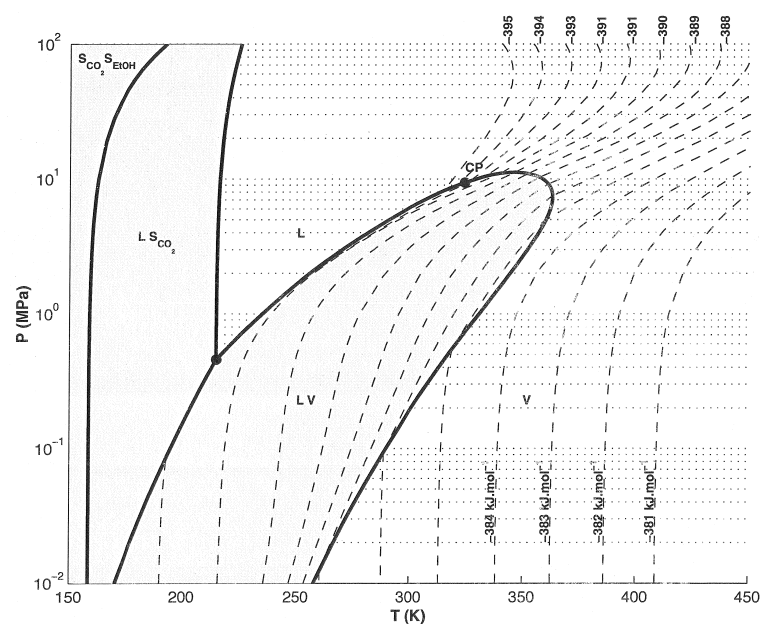

Figure 3. Diagramme température-pression pour un mélange $\mathrm{CO}_{2}$ éthanol. Fractions molaires $95 \% \mathrm{CO}_{2}-5 \%$ éthanol. Calculs effectués avec l'équation d'état de Peng -Robinson (1976).

Dans certains cas, il peut être avantageux de laisser une pression de l'ordre de quelques MPa dans l'enceinte de détente. Certes le niveau de sursaturation en sera réduit, mais la recirculation du fluide deviendra possible. De plus, la chute de pression obtenue reste en général suffisante pour obtenir une chute de la solubilité conséquente. Là encore, le diagramme de Mollier sera d'une grande aide quant à la détermination des conditions expérimentales à utiliser. Enfin, cela permet d'éviter la formation de glace carbonique.

\section{Utilisation d'un co-solvant}

Le procédé RESS présente toutefois un inconvénient majeur souvent signalé (Jung et Perrut, 2001) : de nombreuses molécules ne présentent qu'une très faible solubilité dans le $\mathrm{CO}_{2}$, même à des pressions ou des températures élevées. II est bien sûr envisageable d'utiliser un autre fluide. Cependant, les autres fluides potentiellement utilisables $\left(\mathrm{N}_{2} \mathrm{O}\right.$, alcanes légers,...) sont bien plus dangereux que le $\mathrm{CO}_{2}$ (Raynie, 1993). Une autre possibilité réside dans l'ajout d'un co-solvant (Ekart et al., 1993). Un co-solvant est un troisième composé qui, ajouté en faible proportion au mélange, va permettre dans certains cas une augmentation importante de la solubilité (parfois d'un facteur 100 ou plus). On perd alors l'avantage de n'utiliser aucun solvant organique. Cependant, certains composés comme l'éthanol ou l'acétone de faible toxicité peuvent être utilisés. Le diagramme enthalpique d'un mélange $\mathrm{CO}_{2}+\mathrm{Co}-$ solvant diffère de celui du $\mathrm{CO}_{2}$ pur. La Figure 3 donne par exemple un diagramme $(T, P)$ pour un mélange composé de 95 $\%$ de $\mathrm{CO}_{2}$ et de $5 \%$ d'éthanol (concentrations exprimées en fraction molaire). On constate que l'ajout du co-solvant, même à faible concentration, se traduit par une enveloppe diphasique considérablement plus large. Ceci doit bien sûr être pris en compte dans la détermination des conditions opératoires et de la teneur en co-solvant. Sauceau et al. (2001) montrent par exemple que la solubilité d'un principe actif pharmaceutique dans le $\mathrm{CO}_{2}$ peut être multipliée par 10 avec $1 \%$ de DMSO ou alternativement $5 \%$ d'éthanol. Un autre exemple de l'effet de I'ajout de co-solvant est donné par Chang et Randolph (1989). L'addition de $1,5 \%$ (fraction molaire) de toluène dans l'éthylène supercritique augmente la solubilité du $\beta$-carotène.
Avec ajout de ce co-solvant, la taille et la morphologie des particules obtenues après expansion de la solution sont inchangées. Le choix du co-solvant est crucial et les affinités deux à deux des trois composés doivent être prises en compte. Dans certains cas, un mélange de co-solvants peut être utilisé (Guan et al., 1998). Dans tous les cas, il est souvent nécessaire de procéder à des mesures de solubilité dans le mélange FSC-co-solvant (Sauceau et al., 2000b ; Ting et al., 1993).

Si I'ajout d'un co-solvant permet d'augmenter la solubilité d'un solide, se pose par contre le problème de son élimination. La chute de température induite par la détente peut provoquer dans le pire des cas l'apparition d'une phase liquide majoritaire en co-solvant qui peut dissoudre le solide formé. II est alors d'autant plus nécessaire de disposer de données et d'un modèle permettant la représentation du comportement de mélange multi-constituant et multi-phasique afin de déterminer au mieux les conditions opératoires qui permettent de minimiser la présence de co-solvant résiduel.

\section{Taille et morphologie des poudres obtenues : vers une modélisation du procédé}

La vitesse de nucléation / ou nombre de nuclei formés par unité de temps, est corrélée à la sursaturation absolue $\Delta c$ par la loi de Nyvlt :

$$
J=k \Delta c^{n}
$$

Les paramètres $k$ et $n$ sont déterminés expérimentalement. De très grandes valeurs de sursaturation conduiront donc à un très grand nombre de nuclei. Comme la taille de chaque nucleus est d'autant plus petite que le rapport de sursaturation est grand, le procédé RESS est souvent présenté comme le procédé idéal pour fabriquer des poudres nanométriques. De plus, les conditions uniformes liées à l'onde de choc de la détente induisent une distribution de taille de particules très resserrée. En conséquence, le contrôle de la taille des particules peut $s^{\prime}$ effectuer en modulant la perte de charge $\Delta P$ au niveau de la buse, qui influence directement le niveau de sursaturation. Alessi et al. (1996) ont montré qu'une diminution de la pression en amont et/ou une augmentation de la pression en aval se traduisaient par une augmentation de la taille des particules.

De plus, comme les cristaux continuent de croître dans le jet (Kwauk et Debenedetti, 1993), la taille moyenne des particules obtenues est bien supérieure à celle prédite par la théorie. Ainsi, pour le phénanthrène dans le $\mathrm{CO}_{2}$ supercritique, Debenedetti (1990) indique une taille critique de nuclei de $20 \mathrm{~nm}$ alors que les particules obtenues expérimentalement ont une taille variant de quelques centaines de $\mathrm{nm}$ à quelques $\mu \mathrm{m}$ (Reverchon et Perrut, 2000).

Debenedetti et al. (1993a) ont modélisé les champs de pression, de température et de densité dans un capillaire. Ils ont montré l'influence de la densité sur la taille et la morphologie des particules. Ksibi et al. (1996) ont quant à eux réalisé une simulation numérique du procédé RESS dans laquelle la modélisation de la nucléation du solide est prise en compte couplée à celle de l'hydrodynamique du jet.

Lors de l'étude de polymères dans le $\mathrm{CHF}_{2} \mathrm{Cl}$, Lele et Shine (1992) ont également observé que la densité du fluide au niveau de la buse influençait la morphologie particulaire. Les mêmes auteurs (Lele et Shine, 1994) ont corrélé le type de particules obtenues (fibres ou sphères) avec le temps disponible 
pour la séparation de phases. En effet, pour des polymères, la cristallisation survient à partir d'une phase liquide riche en polymère après une séparation de phases liquide-liquide. De longues fibres sont obtenues pour une séparation de phases en amont de la buse, alors que de fines particules $(0,2$ à $0,6 \mu \mathrm{m})$ ont été produites lorsque la séparation des phases a lieu dans la buse.

Récemment, Helfgen et al. (2001) ont tenté de prédire la formation des particules par simulation numérique. Ils ont étudié la cristallisation de cholestérol, de griséofulvine et d'acide benzoïque à partir de $\mathrm{CO}_{2}$ et de $\mathrm{CHF}_{3}$ comme solvants. Ils ont ainsi observé que la nucléation se produisait dans le jet supersonique, la croissance cristalline étant due principalement à un phénomène de coagulation.

\section{Applications du procédé RESS}

On trouve dans la littérature scientifique un grand nombre d'articles décrivant la réalisation de poudres par le procédé RESS.

En ce qui concerne les poudres minérales, d'autres solvants que le $\mathrm{CO}_{2}$, en particulier l'eau ou le pentane sont assez souvent utilisés. En effet, la plupart des composés minéraux présentent une trop faible solubilité dans le $\mathrm{CO}_{2}$. Matson et al. $(1987 a, 1987 b)$ rapportent la fabrication de poudres et de fibres céramiques à partir de silice et de polycarbosilane. Afin d'obtenir un mélange intime de plusieurs composés céramiques, il est possible de les dissoudre dans l'eau supercritique ( $T C=647 \mathrm{~K}$ et $\mathrm{Pc}=22,4 \mathrm{MPa}$ ) et de détendre la solution afin de provoquer la formation de poudres ultra-fines. Ces poudres peuvent ensuite être frittées, opération d'autant plus aisée que les poudres sont fines et débarrassées de toute impureté. Des particules de sulfure de plomb $\mathrm{PbS}$, un semi-conducteur, ont été obtenues par Sun et al. (2000) à l'aide d'un procédé RESS modifié. En effet, l'expansion est réalisée dans un milieu liquide au sein duquel une réaction chimique a lieu entre un réactif cristallisé par RESS et un autre réactif préalablement dissous dans le liquide. Les solvants utilisés sont en l'occurrence l'ammoniaque, le méthanol et l'acétone.

Cependant, la majorité des études publiées concernent des molécules organiques. On peut trouver quelques exemples d'application dans le domaine des industries agroalimentaires :

- le beurre de cacao, dont les cristaux présentent plusieurs polymorphes (Loisel et al., 1998), est un bon candidat à la micronisation par RESS en raison de sa très grande solubilité dans le $\mathrm{CO}_{2}$ supercritique (Kokot et al., 1999) ;

- le $\beta$-carotène, colorant alimentaire et précurseur de la vitamine $A$, présente lui aussi une bonne solubilité dans le $\mathrm{CO}_{2}$ supercritique (Cygnarowicz, 1990 ; Skerget et al., 1995), le protoxyde d'azote $\mathrm{N}_{2} \mathrm{O}$ et l'éthane (Mendes et al., 1999). De ce fait, sa micronisation par un procédé RESS mérite certainement davantage d'étude que les quelques-unes rapportées jusqu'ici (Chang et Randolph, 1989).

Il faut noter toutefois que les ingrédients et les denrées alimentaires ont généralement une faible valeur ajoutée, ce qui limite leur utilisation par des technologies requérant souvent des investissements élevés. Il en va tout autrement des produits pharmaceutiques, qui ont logiquement fait l'objet d'un plus grand nombre d'études. L'usage du procédé RESS, que ce soit pour produire des particules de substance active ou des systèmes à libération contrôlée avec un polymère, s'est beaucoup développé cette dernière décennie. Plusieurs revues ont fait la synthèse des produits ainsi obtenus (Jung et Perrut,
2001 ; Knutson et al., 1996 ; Tom et Debenedetti, 1991). On trouvera ci-dessous une liste non-exhaustive de quelques-unes de ces substances parmi les plus représentatives (en l'absence de toute autre mention, le solvant utilisé est le $\left.\mathrm{CO}_{2}\right)$ :

- la lovastatine, un hypocholestérolémiant (Mohamed et al., 1989 ; Debenedetti et al., 1993b) ;

- le $\beta$-estradiol, un stéroïde (Krukonis, 1984);

- I'acide salicylique, un antalgique (Domingo et al., 1997) ;

- la caféine et la théophylline (Subra et Debenedetti, 1996);

- le cholestérol (Kröber et al., 1999 ; Helfgen et al., 2001);

- la phénacetine, un analgésique (Loth et Hemgesberg, 1986);

- des hormones : progestérone, testostérone (Jung et Perrut, 2001);

- I'ibuprofène, un anti-inflammatoire (Charoenchaitrakool et al., 2000) ;

- I'a-tocophérol ;

- la griséofulvine, un antibiotique, obtenue par Reverchon et al. (1995) à partir de $\mathrm{CHF}_{3}$ supercritique.

Il faut également garder en mémoire qu'un nombre important de travaux ont été et sont encore effectués par les sociétés pharmaceutiques sans jamais avoir été publiés.

Knutson et al. (1996) ont publié une synthèse de l'utilisation du procédé RESS en vue d'obtenir des micro-sphères polymériques contenant des principes actifs. Ces systèmes, destinés à libérer progressivement la substance active, sont en général composés d'une substance active enrobée ou encapsulée dans un polymère biocompatible. Les polymères les plus utilisés sont I'acide poly-lactique (PLA), I'acide poly-glycolique $(P G A)$ et leurs co-polymères en raison de leur excellente compatibilité après implantation dans le corps humain. Par exemple, la lovastatine, molécule hypocholestérolémiante, a été cristallisée sous forme d'aiguilles enrobées dans des microsphères de DL-PLA (Tom et al., 1992).

Le principe actif et le polymère peuvent être dissous dans le fluide (généralement le $\mathrm{CO}_{2}$ ). Ils peuvent l'être dans le même autoclave ou indépendamment, les deux flux devant alors se rejoindre avant ou au niveau de la buse. Cependant, la plupart des polymères sont peu solubles dans le $\mathrm{CO}_{2}$ ce qui explique que de nombreux co-solvants tels que les fluorohalocarbones et autre CFC aient été utilisés. Malheureusement, la toxicité de ces molécules, ainsi que leur impact négatif sur l'environnement, ont mis un terme probablement définitif à leur utilisation.

\section{Conclusion et perspectives pour le procédé RESS}

Le procédé séduit par sa simplicité qui reste son principal atout. II est potentiellement utilisable avec tout type de molécule et peut en couplage avec un polymère servir à fabriquer des systèmes à libération contrôlée. De plus, les conditions rencontrées lors d'un tel procédé présentent des avantages liés à la fois aux énormes rapports de sursaturation obtenus et au fait que le solvant retourne à l'état gazeux en fin de cycle. Ainsi :

- les particules sont ultra-fines et monodisperses ;

- le fluide est recyclable ;

- il n'y a pas de solvant résiduel ;

- les molécules sensibles à la chaleur, aux chocs thermiques ou mécaniques peuvent être traitées.

Cependant, une limitation importante tient au fait que le procédé RESS n'est pas applicable dans le cas de solutés très (trop) peu solubles. Ceci explique le rapide développement des techniques où le fluide supercritique est utilisé comme anti-solvant. 


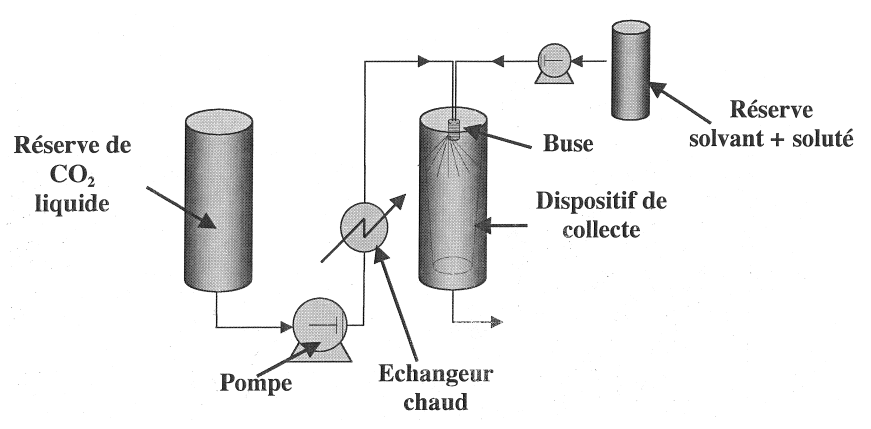

Figure 4. Schéma de principe du procédé SAS en mode semi-continu.

De plus, les articles parus montrent que la taille et la morphologie des particules sont généralement mal contrôlées. Un travail de modélisation incluant les étapes de nucléation et de croissance cristalline dans le jet reste donc à faire. Le rôle de la géométrie de la buse reste également assez flou et demande à être précisé.

II n'en reste pas moins que, pour l'obtention de poudre cristalline ultra-fine, le premier procédé à envisager reste le RESS. Une faible solubilité peut même parfois ne pas être rédhibitoire : en jouant sur la densité du fluide et avec l'ajout éventuel d'un co-solvant bien choisi, I'usage de cette famille de procédés est envisageable, même à une échelle importante.

Enfin, comme cela a déjà été signalé par plusieurs auteurs, le principe du RESS est connu depuis plus d'un siècle ce qui enlève beaucoup de force à l'activité inventive revendiquée dans de nombreux brevets relatifs à ces méthodes.

\section{Les procédés à anti-solvant Principe du procédé}

Quand le soluté est insuffisamment soluble dans le FSC, celui-ci peut être utilisé comme anti-solvant. Ce principe d'utilisation sera particulièrement adapté pour des molécules telles que les sucres, les protéines, les polymères ou les oxydes métalliques connus pour leur très faible solubilité dans le $\mathrm{CO}_{2}$ supercritique. Le terme le plus couramment utilisé pour cette famille de procédés est SAS (pour Supercritical Anti Solvent). Outre le composé à microniser et le FSC, un troisième composé intervient. Il s'agit d'un solvant organique présentant au moins les trois propriétés suivantes:

- être un bon solvant du composé étudié ;

- avoir une bonne compatibilité avec l'anti-solvant. Idéalement, ils doivent même être complètement miscibles ;

- persister le moins possible dans le solide cristallisé à la fin du procédé.

Lors de la mise en contact de la solution avec l'anti-solvant supercritique, celui-ci va se dissoudre dans la phase organique diminuant sa densité, son pouvoir solvant et donc la solubilité du soluté. Concomitamment, le solvant va s'évaporer dans la phase supercritique, augmentant la concentration du soluté. $C^{\prime}$ est ce transfert de masse bi-directionnel qui explique la rapide sursaturation du soluté conduisant à sa nucléation.

Plusieurs façons de mettre en œuvre le procédé SAS ont été décrites :

- la Figure 4 présente un schéma de principe d'un procédé semi-continu où la solution et l'anti-solvant se rejoignent au niveau de la buse d'expansion. Cette méthode est aujourd'hui la plus étudiée car c'est celle qui apparaît comme étant

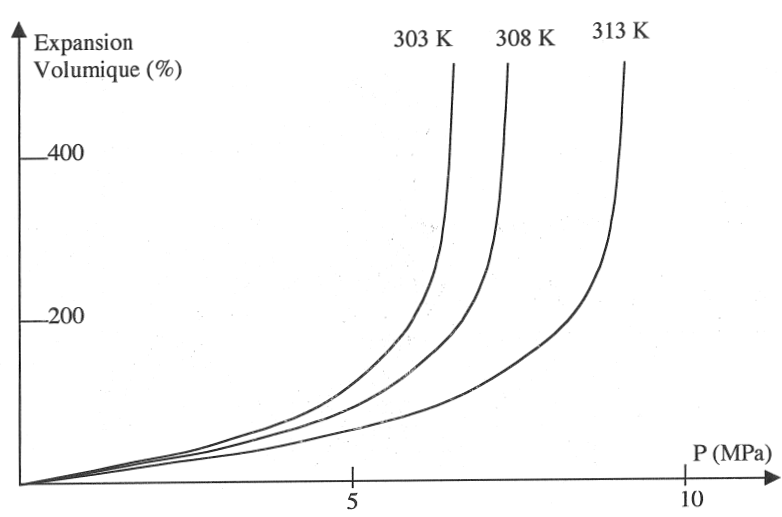

Figure 5. (d'après Reverchon,1999). Expansion volumique du DMSO dans le $\mathrm{CO}_{2}$.

la plus aisément transposable à une échelle industrielle. L'utilisation d'une buse comprenant plusieurs tubes coaxiaux a été revendiquée par Hanna et al. (1998). Cette mise en œuvre a été dénommée SEDS (pour Solution Enhanced Dispersion by Supercritical fluids). À l'exception de la récupération des particules, le reste du procédé peut être opéré en continu. Le solvant peut être récupéré dans des séparateurs en aval, tandis que l'anti-solvant (le plus souvent du $\mathrm{CO}_{2}$ ) peut quant à lui être recyclé par compression et chauffage. Une étape de lavage ou d'extraction au $\mathrm{CO}_{2}$ pur est recommandée en fin de procédé, afin d'éliminer au sein des particules cristallisées les dernières traces de solvant qui pourraient s'y trouver. Ruchatz et al. (1997) ont montré que la quantité résiduelle de solvant est fortement dépendante des paramètres opératoires ;

- le FSC peut être injecté dans un récipient sous pression contenant la solution. Ce procédé discontinu est connu sous I'acronyme de GAS (pour Gaseous Anti Solvent). Ici, le débit $d^{\prime}$ 'injection du fluide supercritique est un paramètre crucial. II provoque une expansion de la solution conduisant à la sursaturation et à la formation des nuclei. Une fois les particules formées, le solvant excédentaire est éliminé par un balayage continu de FSC pur. Les particules sont ensuite collectées après remise à pression atmosphérique de l'enceinte utilisée. Les principaux inconvénients de cette manière de procéder sont liés au mode discontinu : la pression croît continûment tout au long de l'injection du fluide, induisant une sursaturation progressive et non contrôlée et, in fine, à une distribution de taille des particules élargie. Enfin, un passage à l'échelle industrielle semble problématique ;

- à l'inverse du mode précédent, il est possible de disperser la solution organique dans un autoclave où l'anti-solvant a été préalablement pressurisé (Dixon et al., 1993). Le procédé est alors appelé PCA (pour Precipitation with Compressed Antisolvent);

- enfin, la solution peut être pulvérisée sous pression dans un autoclave où l'anti-solvant (le FSC) circule en continu. La dissolution du FSC dans les gouttelettes de la solution provoque la génération des particules, lesquelles seront ensuite récupérées après évacuation du mélange solvant-antisolvant vers des séparateurs et remise à pression atmosphérique du récipient. Ce procédé est parfois noté ASES pour Aerosol Extraction Solvent System (Bleich et al., 1994). 


\section{Optimisation du procédé SAS}

La taille, la distribution de taille et la forme des particules obtenues dépendent fortement des conditions opératoires. Comme pour le RESS, ce sont les températures et pressions en amont comme en aval de la buse qui doivent être contrôlées. Contrairement au RESS, le rôle de la buse n'est pas ici d'assurer une brusque chute de pression, mais plutôt une dispersion optimale sous forme de gouttelettes favorisant les transferts de masse. La géométrie de la buse joue donc un rôle important. Bien entendu, il faut aussi considérer la concentration du soluté dans la solution, les débits mis en œuvre et le rapport des débits entre la solution et l'anti-solvant.

L'expansion volumique du solvant organique due à la rapide dissolution de l'anti-solvant sous pression a souvent été signalée comme étant un paramètre fondamental (Chang et Randolph, 1990 ; Kordikowski et al., 1995). C'est bien sûr particulièrement vrai dans le cas du procédé GAS en mode discontinu. L'équation suivante définit cette expansion :

$$
\Delta V=\frac{V(P, T)-V_{0}}{V_{0}}
$$

$V$ est le volume du liquide expansé à la pression $P$ et la température $T$ et $V_{0}$ est le volume du solvant pur avant l'injection de I'anti-solvant. Une cellule équipée d'une fenêtre en saphir permet de mesurer directement cette expansion volumique. La Figure 5 montre l'expansion du DMSO (un solvant organique fréquemment utilisé) dans le $\mathrm{CO}_{2}$ en fonction de la pression. Pour une même température, le volume du liquide croît avec la pression. Cependant pour une pression donnée, l'expansion volumique décroît quand la température augmente. Warwick et al. (2000) ont corrélé avec un autre solvant, le DMF, cette expansion avec la fraction molaire du $\mathrm{CO}_{2}$. Ils ont montré que toutes les isothermes se ramènent à une seule courbe de forme semblable à celles de la Figure 5. Pour un solvant donné, l'expansion volumique ne dépend donc que de la fraction molaire des constituants du mélange. Cette expansion volumique peut également être prédite à l'aide d'une équation d'état et des règles de mélange classiques. L'équation d'état la plus utilisée est celle de Peng-Robinson (1976), même si de nombreuses autres l'ont été. Reverchon (1999) a synthétisé I'ensemble des études où une telle expansion a été mesurée en fonction des conditions opératoires et de la nature du solvant. Il faut noter que ces observations sont surtout utiles lorsque des particules sont produites à partir d'une phase liquide riche en solvant. De la Fuente Badilla et al. (2000) ont travaillé avec des données concernant plusieurs mélanges ternaires anti-solvant/solvant/soluté dont le mélange $\mathrm{CO}_{2}$-toluènenaphtalène. En utilisant une nouvelle définition de l'expansion, qui fait intervenir le volume molaire du solvant, ils ont montré que les courbes d'expansion passent par un minimum correspondant à la cristallisation de la phase solide. Ceci permet donc de choisir les conditions opératoires optimales d'un procédé GAS.

En vue d'une industrialisation du procédé, il ne fait pas de doute que c'est le mode semi-continu avec co-injection de la solution et de l'anti-solvant qui est le plus prometteur. Dans ce cas, les courbes d'expansion deviennent moins pertinentes dans la mesure où ce sont d'autres conditions qui peuvent présider à la génération des particules. En effet, celles-ci sont formées à partir des gouttes de la solution qui sont pulvérisées dans la phase supercritique. Ainsi, connaissant les débits relatifs (celui d'anti-solvant est souvent supérieur de 10 à 1000 fois à celui de la solution), un diagramme de phases pression-température ou pression-enthalpie peut s'avérer fort utile pour connaître le nombre des phases en présence et choisir les conditions opératoires. La Figure 3 donne un exemple d'un tel diagramme dans le cas du mélange composé de $95 \%$ de $\mathrm{CO}_{2}$ et de $5 \%$ d'éthanol (pourcentages exprimés en fraction molaire). Ces valeurs correspondent à un rapport des débits de l'ordre de 20, qui est du même ordre de grandeur que ceux souvent utilisés expérimentalement. On distingue clairement l'enveloppe où coexistent une phase liquide et une phase vapeur, zone que l'on cherche souvent à éviter afin de ne pas avoir apparition d'une phase liquide dans la chambre d'expansion. Une telle phase pourrait en effet re-solubiliser les particules néoformées.

\section{Mécanismes de formation des particules}

Dans les procédés semi-continus, qui sont les plus étudiés en raison de leur potentiel d'industrialisation, le transfert de masse et la nucléation se déroulent à la surface des gouttelettes de solution dans un flux d'anti-solvant. Il est donc nécessaire de comprendre quel est le devenir de ces gouttelettes au cours du procédé. Deux phénomènes simultanés entrent en compétition :

- I'effet anti-solvant, c'est-à-dire la dissolution de l'anti-solvant dans la solution. Il s'ensuit un gonflement des gouttelettes;

- l'évaporation du solvant dans l'anti-solvant. Ce phénomène induit une réduction de la taille des gouttelettes.

On peut donc se demander lequel de ces deux phénomènes prédomine. En d'autres termes, les gouttes de solution dans le courant d'anti-solvant gonflent-elles ou se réduisent-elles ? II faut également prendre en compte le fait qu'au-delà du point critique du mélange, le système tend vers un équilibre thermodynamique monophasique. À l'approche de cet équilibre, il devient donc impropre de parler de gouttes. Shekunov et al. (2000a) ont récemment publié des résultats obtenus par des méthodes optiques : analyse d'images, interférométrie laser et vélocimétrie par imagerie de particules (PIV). En étudiant le mélange binaire éthanol/ $\mathrm{CO}_{2}$ ils ont montré que les gouttes d'éthanol dans un flux turbulent de $\mathrm{CO}_{2}$ ne gonflent pas, comme on aurait pu s'y attendre, mais au contraire ont tendance à diminuer de taille au fur et à mesure que $I^{\prime}$ éthanol se dissout (s'évapore) dans le $\mathrm{CO}_{2}$. De plus, selon la valeur de la pression (en deçà ou au-delà du point critique du mélange), le comportement des gouttes change. Aux pressions inférieures à celle du point critique de mélange, les gouttes d'éthanol décroissent régulièrement en taille. La nucléation et la croissance cristalline se produisent à leur surface pour donner des particules agglomérées. Pour des pressions plus élevées, un jet liquide d'éthanol sort de chaque gouttelette. La nucléation se produit à l'intérieur des gouttelettes, qui se rétrécissent, et le régime fluide dans lequel se passe l'opération a une grande importance. Aucun gonflement n'a été observé par ces auteurs.

Reverchon (1999) a noté que la morphologie des particules était liée aux volumes d'expansion $\Delta V$. Faisant la synthèse de nombreuses expériences effectuées en batch (procédé GAS), il a constaté que de faibles $\Delta V$ conduisent à des particules agglomérées. Ces particules proviennent d'une cristallisation au sein de la phase liquide. Des valeurs moyennes de $\Delta V$ donnent des particules en forme de sphères creuses, appelées ballons, suggérant un mécanisme de cristallisation à la surface de gouttelettes en expansion rapide. Enfin, c'est pour les plus 
hautes valeurs de $\Delta V$ que les particules les plus petites avec une distribution de taille resserrée sont obtenues. Dans ce cas, le mécanisme proposé est une désintégration des sphères creuses bien qu'une cristallisation à partir de gouttelettes implosant soit aussi possible. Cependant, il ne faut pas mésestimer la contribution des phénomènes additionnels, comme la croissance ou la coalescence, qui peuvent ensuite grandement modifier la morphologie granulaire. Que ce soit en mode discontinu ou semi-continu, le temps de présence des particules dans un fluide composé de $\mathrm{CO}_{2}$ et d'un solvant n'est pas sans importance. Le solvant peut en effet également se comporter comme un co-solvant et entraîner des phénomènes de dissolutionrecristallisation et, bien sûr, des pertes de rendement.

\section{Modélisation des procédés SAS}

La modélisation des procédés de type SAS repose bien évidemment sur les calculs thermodynamiques d'équilibres entre phases. Cependant, dans le cas d'une mise en œuvre semi-continue, cette approche est insuffisante. Ainsi, en présence de très hauts niveaux de sursaturation, la cristallisation se déroule loin de l'équilibre et la génération des particules ne peut être prédite, ni même décrite, à partir de la seule thermodynamique. De plus, les considérations cinétiques, notamment l'hydrodynamique du jet en sortie de buse, la cinétique de nucléation (primaire et secondaire) et la croissance des cristaux doivent aussi être prises en compte. Un modèle complet devrait donc idéalement comprendre quatre volets :

- thermodynamique : connaissance et description du mélange binaire solvant-anti-solvant, permettant le choix du couple $(P, T)$. La dissolution de I'anti-solvant dans le solvant, ainsi que l'évaporation du solvant dans l'anti-solvant, peuvent ainsi être contrôlés.

- hydrodynamique : la géométrie du jet à la sortie de la buse et la manière dont il va être dispersé (relié au champ de vitesses et à la turbulence) conditionnera la formation des gouttes. Les tensions de surface et les viscosités des fluides doivent être intégrées au modèle ;

- cristallisation : le transfert de masse bi-directionnel (du solvant vers l'anti-solvant et vice-versa) doit pouvoir être simulé pour connaître l'évolution des rapports de sursaturation et la vitesse de production des nuclei ;

- thermique : prise en compte à l'échelle microscopique, des transferts thermiques. Par exemple, lorsque le soluté précipite, il transfère au milieu sa chaleur latente de changement de phase induisant des modifications locales de densité.

Lora et al. (2000) ont proposé un modèle d'un tel procédé SAS avec le $\mathrm{CO}_{2}$ comme anti-solvant, le toluène comme solvant, le naphtalène et le phénanthrène comme solutés. Ils ont pris en compte trois des volets déjà cités : équilibres de phases, hydrodynamique et transfert de masse. À partir de quelques hypothèses simplificatrices, ils proposent une intéressante simulation du procédé SAS dès lors que I'on connaît les équilibres entre phases du mélange binaire solvant-anti-solvant. Leur simulation montre que les gouttelettes subissent un rapide gonflement suivi d'une phase plus lente de rétrécissement. La dissolution du $\mathrm{CO}_{2}$ dans le solvant et l'évaporation du solvant dans le $\mathrm{CO}_{2}$ augmentent avec la solubilité du $\mathrm{CO}_{2}$ dans le solvant. Trois solvants ont ainsi pu être comparés : le toluène, I'acétone et le DMSO. De plus, les phénomènes de cristallisation des deux solutés testés varient : I'effet anti-solvant est suffisant pour provoquer la cristallisation du phénanthrène, alors que l'évaporation du solvant est impliquée dans la cristallisation du naphtalène. La variation du rapport des débits solvant/anti-solvant est donc importante pour le naphtalène alors qu'elle est sans effet sur le phénanthrène.

Werling et Debenedetti (1999) ont également étudié le système $\mathrm{CO}_{2}$-toluène (en l'absence de soluté à cristalliser). Par modélisation numérique du transfert de masse bi-directionnel entre le $\mathrm{CO}_{2}$ et le toluène, ils ont montré qu'aux conditions sous-critiques, les gouttelettes subissent successivement une phase de gonflement suivie d'une phase d'effondrement. Les mêmes auteurs dans un second article (Werling et Debenedetti, 2000) ont mis en évidence la dépendance du devenir des gouttelettes en fonction des densités relatives du solvant et de I'anti-solvant. Si ce dernier est moins dense que le solvant - cas le plus fréquent - les gouttelettes peuvent gonfler. Dans le cas contraire, elles rétrécissent. Le degré de gonflement ou de rétrécissement, ainsi que la durée de vie des gouttelettes, sont très sensibles aux variations des conditions opératoires. Finalement, pour optimiser la production des particules, notamment lorsqu'on cherche à fabriquer de très fines particules, il semble avantageux de travailler dans la zone monophasique située au-delà du point critique de mélange. Dans cette zone de hautes pressions, les transferts de masse et les degrés de sursaturation sont plus élevés. La durée de vie moyenne d'une goutte est donc minimisée en raison du fort transfert massique et conduit à la production de très petites particules. Ces auteurs concluent que pour mettre en œuvre un procédé SAS semi-continu, il est au préalable nécessaire d'étudier les équilibres entre phases du mélange binaire solvant/anti-solvant de façon à pouvoir éviter la zone à proximité du point critique.

Plus récemment, le groupe de recherches de Bradford (Shekunov et al., 2001) a étudié l'effet des conditions de mélange sur la cristallisation du paracétamol à partir d'une solution éthanolique dans le $\mathrm{CO}_{2}$ supercritique. Pour des nombres de Reynolds élevés ( $\operatorname{Re}>10^{4}$ ) dans la buse, ils constatent un bon micromélange, conduisant à des petits diamètres de particules. En effet, dans le jet, la nucléation entre en compétition avec le mélange. Des données expérimentales et d'autres issues du calcul numérique sont données. La taille des particules décroît rapidement quand Re augmente jusqu'à atteindre $10^{4}$. Elle passe alors par un minimum, avant de présenter une certaine augmentation attribuée à un plus faible temps de séjour dans la zone à forte sursaturation. La taille des particules est aussi fonction du diamètre de la buse utilisée. Les auteurs concluent sur la nécessité d'un travail supplémentaire sur la géométrie des buses et sur les cinétiques de cristallisation avant de pouvoir proposer un modèle complet capable de prédire avec précision la distribution de taille des particules ainsi fabriquées.

\section{Contrôle du polymorphisme cristallin}

La plupart des molécules organiques que l'on cherche à cristalliser dans l'industrie pharmaceutique présentent de nombreux groupes polaires. Selon la manière dont les molécules vont s'associer les unes aux autres, plusieurs polymorphes cristallins pourront être obtenus. Pour le pharmacien, deux polymorphes sont deux substances différentes, et il est donc de la plus haute importance de savoir obtenir de façon reproductible, le polymorphe recherché. Ceci est vrai aussi dans d'autres secteurs : ainsi, il est bien connu qu'une « mauvaise » re-cristallisation du beurre de cacao dans le chocolat explique le phénomène indésirable appelé blanchiment gras (Beckett, 2000). Les techniques de cristallisation classiques 


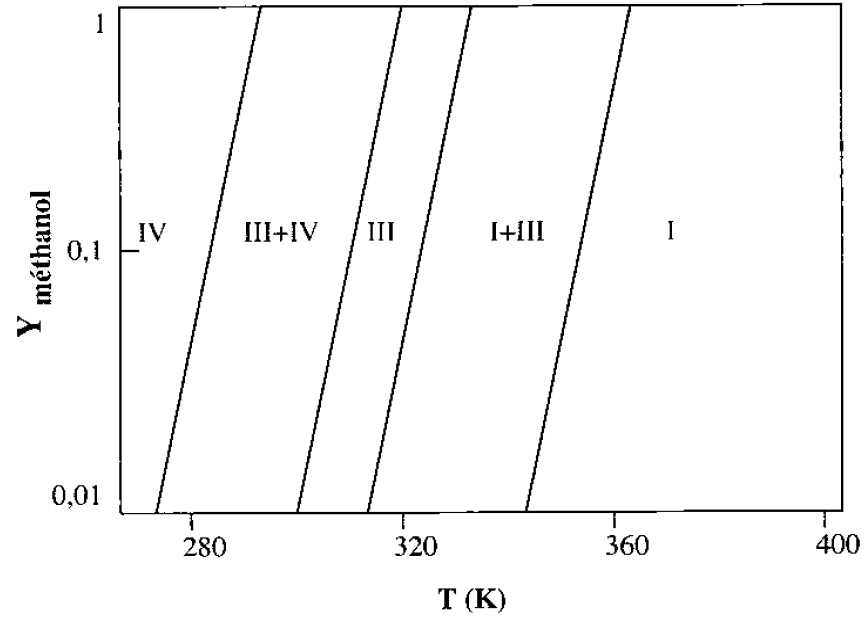

Figure 6. Obtention des polymorphes du Sulfathiazole en fonction de $T$ et de la fraction molaire de solvant (d'après Kordikowski et al. 2000).

conduisent souvent à un mélange des différents polymorphes, en raison de la longueur et de la complexité des procédés comportant plusieurs opérations unitaires. Une des caractéristiques les plus prometteuses des procédés supercritiques réside dans le fait que ces procédés à une seule étape peuvent permettre de contrôler le polymorphisme cristallin.

Kordikowski et al. (2000) ont travaillé en SAS semi-continu avec le sulfathiazole, composé présentant cinq polymorphes différents. Avec le méthanol comme solvant et le $\mathrm{CO}_{2}$ comme anti-solvant, ils ont montré l'importance du débit de méthanol et de la température opératoire pour obtenir un polymorphe pur. Ainsi, en choisissant correctement la température, trois polymorphes purs, notés I, III et IV, ont pu être obtenus. Le rapport des débits méthanol/ $\mathrm{CO}_{2}$ a quant à lui moins d'influence que la température, comme le montre la figure 6 . Cependant, il permet le contrôle des tailles de particule.

Hanna et al. (1998), ont obtenu l'un ou l'autre des deux polymorphes de Xinafoate de Salmeterol (médicament anti-asthme) avec une grande pureté en fonction des conditions opératoires, température et pression. Dans notre laboratoire, des résultats non encore publiés viennent confirmer qu'un polymorphe donné peut être obtenu avec le procédé SAS, alors que la cristallisation en milieu liquide ne permet pas d'atteindre ce résultat. Les conditions opératoires, mais aussi la cristallisation conjointe d'un autre composé, jouent un rôle dans ce domaine.

Un anti-inflammatoire non stéroïdien, l'indiméthacine, a été micronisé avec le DMF comme solvant par un procédé GAS (Warwick et al., 2000). Deux polymorphes cristallins ont été obtenus selon la vitesse d'expansion. Lorsqu'on opère plus lentement, des cristaux rhombiques sont obtenus, alors qu'un mélange de cristaux rhombiques et de cristaux bi-pyramidaux provient d'une expansion plus rapide. Ces auteurs ont également noté une diminution de la taille des cristaux lorsque la vitesse d'expansion augmente.

\section{Les applications des procédés SAS}

Les procédés qui utilisent un fluide supercritique comme anti-solvant peuvent trouver des applications dans de très nombreux secteurs industriels. On trouvera ci-dessous quelques exemples représentatifs publiés récemment.
Les explosifs, ne pouvant à l'évidence pas être broyés, furent parmi les premières substances testées par ces procédés. Dans ce cas, c'est une densité maximale qui est recherchée. Des microparticules dépourvues de toute porosité ont ainsi pu être obtenues. Gallagher et al. (1992) ont travaillé avec des explosifs tels que le RDX, le HMX et la nitroguanidine. Ils ont observé des formes de particules différentes selon la vitesse d'addition de I'anti-solvant.

Des produits minéraux, comme des superconducteurs ou des catalyseurs, ont également été testés. Reverchon (1999, 2002) a publié plusieurs résultats concernant des précurseurs de superconducteurs et de catalyseurs.

Des produits d'origine végétale, comme la lécithine de soja ou I'huile de graines de coriandre, ont pu être fractionnés avec des procédés de type SAS avec I'hexane comme solvant (Catchpole et al., 1996). La cristallisation de lécithine de soja à partir de solution alcoolique a également été décrite (Magnan et al., 2000), alors que du jaune d'œuf a été cristallisée avec un mélange d'hexane et d'éthanol par Weber et al. (1999).

Les molécules de l'industrie pharmaceutique présentent généralement la double caractéristique d'être à haute valeur ajoutée et d'avoir une très faible solubilité dans l'eau. Cette dernière propriété constitue, bien entendu, un gros inconvénient pour rendre le principe actif bio-disponible dans l'organisme du patient. La technologie des fluides supercritiques, qui permet notamment d'améliorer grandement la surface spécifique des poudres, apporte des réponses originales à ce problème et a été très largement testée sur des molécules pharmaceutiques depuis une dizaine d'années. Les procédés à anti-solvant ont été les plus étudiés car on peut presque toujours trouver un solvant quelle que soit la molécule à microniser.

Les protéines représentent un cas particulier qui a fait l'objet de plusieurs études. Le développement du génie génétique a permis I'apparition de nouvelles protéines thérapeutiques, telles que l'insuline recombinante humaine. Cependant, une formulation sèche de telles molécules reste difficile, notamment en raison de la préservation de la bioactivité. Là encore, les procédés de type SAS présentent des avantages, notamment en comparaison à l'atomisation qui met en jeu de hautes températures ou à la lyophilisation, souvent jugée trop onéreuse.

Un groupe de recherches à l'université de Princeton a publié des travaux concernant diverses enzymes et protéines : insuline, catalase et peroxydase (Yeo et al., 1993). Plusieurs morphologies cristallines furent obtenues. De l'insuline ayant conservé toute son activité biologique a même été obtenue sous forme de particules sphériques, à partir de solution de DMSO et de DMF. Par le biais de la spectroscopie RAMAN, Winters et al. (1996) ont montré, avec $\mathrm{I}^{\prime}$ insuline, la trypsine et le lysozyme, que des modifications des conformations spatiales peuvent être induites selon les conditions expérimentales. L'insuline est la protéine la plus sujette à de tels changements qui, toutefois, ne modifient pas son activité biologique.

Avec la trypsine et le lysozyme, Sloan et al. $(1998,1999)$ ont obtenu de très fines particules (diamètre moyen de $0,8 \mu \mathrm{m}$ pour le lysozyme et $1,5 \mu \mathrm{m}$ pour la trypsine) avec une distribution de taille resserrée. Cependant, alors qu'une activité biologique totale était conservée pour le lysozyme, seulement $36 \%$ de celle-ci a pu être récupérée avec la trypsine. Ces auteurs indiquent qu'un changement dans la structure tri-dimensionnelle de la protéine pourrait expliquer ce résultat. 
Les antibiotiques constituent eux aussi une classe de molécules qui a été étudiée du point de vue de la micronisation. Reverchon et al. (1999) ont montré qu'avec la N-méthyl 2-pyrolidone comme solvant, la tétracycline et I'amoxycilline pouvaient être micronisées, contrairement à l'ampicilline. Avec le DMSO, aucun de ces trois antibiotiques n'a pu l'être.

On trouve encore dans la littérature de nombreux autres principes actifs ayant été traités par des procédés de type SAS. On peut citer, par exemple, l'acide p-hydroxybenzoïque (Thiering et al., 1998 ; Sze Tu et al., 1998) et le salmeterol xinafoate, une molécule active contre l'asthme (Hanna et al., 1998). Jung et Perrut (2001) ont récemment recensé une liste des composés ayant été testés par toute la famille des procédés SAS-GAS-ASES-SEDS-PCA.

La micronisation de polymères a aussi été décrite. Dixon et al. (1993) ont étudié la formation de micro-sphères de polystyrène. Mais c'est bien sûr la famille des polymères biodégradables, que sont I'acide poly-lactique (PLA) I'acide poly-glycolique (PGA) et leurs co-polymères (PLGA), qui a été la plus étudiée. Bleich et al. (1994) ont par exemple obtenu des micro-particules de PLA avec le chlorure de méthylène comme solvant.

Il faut également observer que les excipients, qui sont souvent le composé majoritaire d'un comprimé pharmaceutique, ont eux aussi fait l'objet d'études. C'est le cas notamment de di-holosides comme le maltose, le lactose ou le saccharose, quasiment insolubles dans de nombreux solvants organiques mais présentant en revanche une grande solubilité dans l'eau. Cependant, l'eau n'est que très faiblement soluble dans le $\mathrm{CO}_{2}$ supercritique, qui reste l'incontournable anti-solvant. Pour s'affranchir de ce problème, Palakodaty et al. (1998a, 1998b) proposent une buse à trois conduits co-axiaux, permettant d'injecter simultanément trois fluides : I'anti-solvant supercritique, la solution aqueuse de sucre et la solution organique contenant le principe actif. Ainsi, la molécule active et son excipient sont co-cristallisés. Ce système présente en outre l'avantage de pouvoir éliminer l'eau résiduelle avec le solvant organique, dans la mesure où ils présentent une bonne solubilité mutuelle. De nombreux autres exemples de co-cristallisation ont été publiés avec ce type de procédé. On en trouvera quelques-uns ci-dessous dont le but souvent recherché est la réalisation d'un système à relargage différé :

- la carmabazépine, utilisée dans le traitement de l'épilepsie et des psychoses maniaco-dépressives, a été co-cristallisée avec du PEG. En utilisant de l'éthyl acetate et de l'acétone comme solvants, Kikic et al. (2000) ont obtenu des micro-particules présentant une vitesse de dissolution améliorée ;

- deux autres molécules, l'acétaminophène (paracétamol) et son précurseur la p-acétoxyacétanilide, ont été co-cristallisées (Shekunov et al., 2000b). Une incorporation des deux composés a été obtenue sous la forme d'une solution solide, la structure cristalline de l'acétaminophène variant avec le taux $d^{\prime}$ incorporation du précurseur ;

- Bertucco et al. (1996) ont étudié la co-cristallisation de l'ester benzylique de l'acide hyaluronique (HYAFF) avec trois principes actifs, une protéine et deux stéroïdes. Ils ont obtenu le principe actif encapsulé dans des particules sphériques submicroniques de polymère amorphe. Cependant la solubilité non négligeable de deux des molécules actives dans l'anti-solvant $\left(\mathrm{CO}_{2}\right)$ affecte négativement le rendement de l'opération ;

- Sze Tu et al. (1998) ont co-cristallisé l'acide p-hydroxybenzoique avec des polymères bio-compatibles, le PLA et le PLGA, avec comme solvants l'acétone, le chlorure de méthylène et le méthanol. La taille des microparticules obtenues (diamètre moyen inférieur à $2 \mu \mathrm{m}$ ) était une fonction décroissante de la pression pour le PLGA, alors que l'inverse a été observé pour le PLA.

\section{Conclusion et perspectives pour le procédé SAS}

Les procédés à anti-solvant de type SAS sont l'objet d'un intérêt croissant qui ne se dément pas depuis une dizaine d'années. Si, au début des années 90, un plus grand nombre d'articles relatifs au procédé RESS ont été publiés, la tendance s'est inversée durablement au profit du SAS. L'intérêt majeur de ce procédé est bien sûr son utilisation potentielle avec n'importe quelle molécule. II est en effet presque toujours possible de trouver un couple solvant-anti-solvant convenable. Comme I'anti-solvant est en général fixé (le $\mathrm{CO}_{2}$ est présent dans plus de $95 \%$ des applications), le choix du solvant est déterminant. Il doit être un bon solvant de la molécule à cristalliser et être miscible, au moins partiellement, avec le $\mathrm{CO}_{2}$. De nombreux solvants classiques comme l'éthanol, le toluène et l'acétone. sont miscibles avec le $\mathrm{CO}_{2}$. Cependant, lorsque le solvant choisi est une plus grosse molécule, le cas diffère et la quantité de $\mathrm{CO}_{2}$ qu'il est possible de dissoudre dans le solvant peut être un facteur limitant. En d'autres termes, l'effet anti-solvant peut s'avérer insuffisant pour assurer une nucléation efficace. II faut cependant noter que, moins la molécule étudiée est soluble dans l'anti-solvant, plus facile sera la génération de particules. De plus, les problèmes de perte de matière et de baisse du rendement global du procédé seront minimisés. Une fois le co-solvant choisi, deux autre paramètres sont cruciaux : le rapport des débits des fluides et la géométrie du dispositif au sein duquel le mélange des fluides va s'opérer.

Finalement, des micro-particules ont été obtenues avec une grande variété de composés : explosifs, polymères, protéines, antibiotiques, ... Leur forme varie depuis des sphères creuses (Reverchon, 1999), en passant par des sphères pleines, ou des fibres (Dixon et Johnston, 1991). Des travaux récents en modélisation ont permis de faire progresser significativement la compréhension (Werling et Debenedetti, 1999 , 2000). Malgré tout, l'influence des conditions opératoires sur le type de particules obtenues est loin d'être encore totalement élucidée.

\section{PGSS, micro-encapsulation par les FSC et autres procédés Le procédé PGSS}

PGSS est un acronyme signifiant Particle generation from Gas Saturated Solutions. Un gaz sous pression peut être solubilisé dans un liquide en grande quantité : c'est cette propriété qui est utilisée. Un gaz dense, le plus souvent il s'agit de $\mathrm{CO}_{2}$, est dissous dans un liquide dans un premier autoclave. Ce liquide peut être une solution, une suspension ou encore un solide fondu.

La solution saturée en gaz ainsi obtenue est envoyée dans une chambre d'expansion à travers une buse (voir Figure 7). La génération de particules a lieu lors de la détente et les particules formées peuvent ensuite être récupérées dans l'autoclave d'expansion.

Ce procédé a surtout été développé pour des polymères dans lesquels de grandes quantités de $\mathrm{CO}_{2}$ peuvent être dissoutes. Cette dissolution se traduit généralement par des modifications dans les températures de transition vitreuse et de 


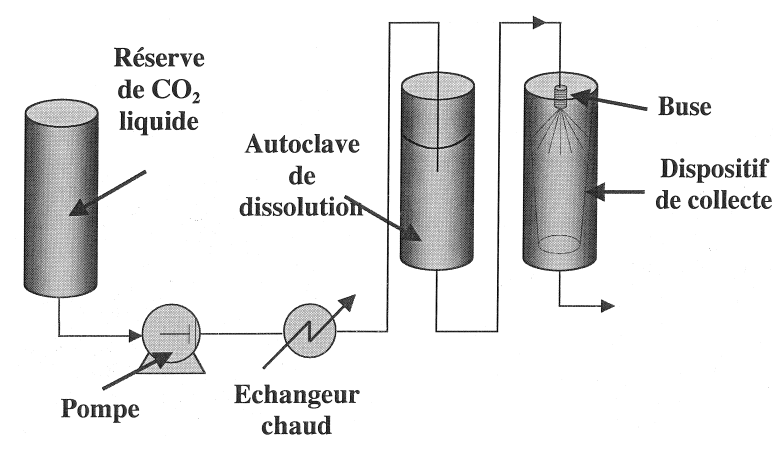

Figure 7. Schéma de principe du procédé PGSS.

fusion, ainsi que dans la densité du polymère. Lorsqu'un tiers corps a préalablement été dissous dans le polymère, des micro-sphères de polymère enrobant la substance considérée peuvent être obtenues après dépressurisation.

À la différence des procédés RESS et SAS, le $\mathrm{CO}_{2}$ est parfois utilisé pour sa faculté à réduire la viscosité, ce qui va faciliter le transport et la manutention de ces solutions. Un procédé industriel a d'ailleurs été développé sur ce principe. II s'agit du procédé Unicarb $®$, qui a fait l'objet de plusieurs brevets (Jung et Perrut, 2001) déposés par Union Carbide (Van den Bos et al., 1996). Ce procédé, appliqué pour des peintures, des vernis et d'autres produits de revêtement, n'est pas à proprement parler un procédé de génération de particules mais plutôt de génération de gouttelettes. II est à noter qu'il permet une réduction considérable des rejets liquides, qui restent un problème majeur dans l'industrie des peintures.

Knez (2000) a publié une revue de l'utilisation de ce procédé pour la production de particules. Des polymères (Weidner et al., 1996), plusieurs types de lipides et deux médicaments, la nifédipine et la félodipine, ont ainsi été micronisées avec succès.

Un autre procédé de génération de particules proche du PGSS a été décrit par Sievers et al. (2000). Il s'agit de la production d'un aérosol qui est ensuite séché. Ce procédé conduit à de très fines particules, utilisables dans des formulations pharmaceutiques pour un traitement par inhalation. $\mathrm{Du} \mathrm{CO}_{2}$ supercritique est mis en contact avec une solution aqueuse $d^{\prime} u n$ principe actif. Cette mise en contact est réalisée dans un té comportant une restriction de diamètre en sortie, laquelle va jouer le rôle d'une buse de dépressurisation. Les microgouttes ainsi produites sont séchées dans un courant d'azote chaud. Ce procédé breveté a été utilisé avec de l' $\alpha$-lactose, excipient classiquement utilisé dans les préparations à inhaler. Deux principes actifs contre l'asthme, le sulfate d'albuterol et le cromolyne de sodium, ont également été cristallisés. Les particules obtenues, de forme sphérique, sont de très petites tailles, avec un diamètre moyen compris entre 0,1 et $3 \mu \mathrm{m}$, taille compatible avec une formulation pour inhalation.

\section{Micro encapsulation et enrobage}

La micro-encapsulation et l'enrobage peuvent être réalisés avec des procédés de type RESS, SAS et PGSS. II s'agit d'un champ $d^{\prime}$ investigation très large car de nombreux secteurs industriels sont à la recherche de systèmes à relargage différé. On peut citer sans être exhaustif la pharmacie, l'agrochimie, la cosmétique, les encres et peintures, etc. Le choix du procédé dépend de nombreux paramètres, au premier rang desquels vont se trouver la solubilité de la substance à encapsuler et le type de polymère.
La plupart du temps, une co-cristallisation est réalisée, conduisant selon les cas à des micro-sphères, des micro-capsules ou encore des liposomes. Avec le PLA, relativement soluble dans le $\mathrm{CO}_{2}$, le procédé RESS est souvent choisi, comme par exemple avec le naproxène dans les travaux de Kim et al. (1996). Pour des polymères présentant des solubilités dans le $\mathrm{CO}_{2}$ plus faibles, comme les PEG, le RESS est encore utilisable avec I'adjonction de co-solvant. Mishima et al. (2000) ont ainsi encapsulé plusieurs protéines dans le PEG. Par contre, pour les polymères les moins solubles dans le $\mathrm{CO}_{2}$, on va trouver surtout des procédés de type SAS, comme indiqué plus haut.

L'enrobage de particules pré-existantes a aussi été décrit. Par exemple, Richard et al. (2000) ont déposé par simple détente un film de polymère, préalablement solubilisé dans le $\mathrm{CO}_{2}$, sur des particules de paracétamol. Tsutsumi et al. (1995) ont eu l'idée d'associer la technologie du lit fluidisé avec le RESS pour enrober des particules de catalyseur par de la paraffine.

\section{Réactions chimiques conduisant à des particules}

Ce principe a surtout été appliqué à des composés minéraux, comme les métaux, les oxydes métalliques et les céramiques. Des poudres de titane, d'aluminium et d'oxydes de magnésium peuvent être obtenues par décomposition thermale d'un précurseur en milieu alcoolique supercritique (Chhor et al., 1992 , 1995). Les mêmes auteurs ont proposé un procédé continu de production d'oxyde de titane (Chhor et al., 1994). De très petites particules (200 à $300 \mathrm{~nm}$ ) ont été obtenues avec une productivité de $20 \mathrm{~g} \cdot \mathrm{h}^{-1}$.

Avec le $\mathrm{CO}_{2}$ supercritique comme milieu réactionnel, Papet et al. (2000) ont réalisé la réaction de l'eau avec un précurseur organo-métallique. En ajoutant l'eau à une solution d'alkoxyde de titane dans le $\mathrm{CO}_{2}$, ils ont obtenu des particules d'hydroxyde de titane et de dioxyde de titane. La taille (500 à $600 \mathrm{~nm}$ ) et la surface spécifique $\left(>400 \mathrm{~m}^{2} \cdot \mathrm{g}^{-1}\right)$ des particules obtenues sont particulièrement remarquables.

\section{Applications et stratégies industrielles}

Comme nous l'avons vu tout au long de cet article, de nombreux exemples d'application sont disponibles dans divers domaines industriels. II semble pourtant que ce soit l'industrie pharmaceutique qui soit la plus concernée par le développement de ces technologies (Morris et al., 1998).

Dans les industries de la santé, peu de procédés utilisant les FSC ont jusqu'ici donné lieu à des produits commerciaux. Seul le traitement du tissu osseux par $\mathrm{CO}_{2}$ supercritique (Fages et al., 1994) a conduit à la mise sur le marché en Europe d'un substitut osseux d'origine bovine par la société Depuy-Bioland. Ce traitement permet en outre d'assurer une sécurité virale inconnue jusqu'alors pour ce type de greffons (Fages et al., 1998a, 1998b).

Dans le domaine du médicament, les FSC devraient permettre d'aider à la résolution de problèmes devenus aujourd'hui très aigus. En effet, lorsqu'un médicament est injecté, un pic d'activité apparaît dès les premières heures, alors qu'il peut être souhaitable d'étaler l'effet thérapeutique sur plusieurs jours ou davantage. Les systèmes d'encapsulation à relargage différé trouvent donc là une de leurs principales applications.

À l'inverse, de nombreuses molécules actives, qui rentreront dans la composition des médicaments de demain, sont pour beaucoup d'entre elles quasiment insolubles dans l'eau. Or, pour atteindre leur cible, ces molécules doivent passer du tractus gastro-intestinal au flux sanguin afin d'être transportées 
sous forme dissoute par le plasma sanguin jusqu'à l'organe cible. Dans le cas d'une administration non orale, telle que la voie pulmonaire ou l'injection intra-veineuse, la solubilité dans les fluides biologiques reste indispensable. La bio-disponibilité du principe actif dépend donc en premier lieu de sa vitesse de dissolution. Si celle-ci est trop faible, une dose plus importante devra être employée, avec tous les risques que cela comporte. Compte tenu de la variabilité biologique intrinsèque à toute espèce vivante, il est essentiel que la dose active soit la plus faible possible afin de prévenir tout accident chez les patients les plus réceptifs. Un verrou technologique majeur à faire sauter est donc de rendre plus solubles ces molécules. Dans la mesure où les procédés décrits dans cet article sont susceptibles d'apporter des réponses concrètes à ce problème majeur, il est évident que I'industrie pharmaceutique s'intéresse de près à ces nouveaux procédés. Schématiquement, deux stratégies peuvent être employées :

- augmenter la vitesse de dissolution, généralement par micronisation conduisant à une augmentation de la surface spécifique ;

- augmenter la solubilité, ce qui suppose de masquer le principe actif par micro-encapsulation pour former un complexe plus soluble que ne l'était la molécule originelle.

La première consiste à générer de plus petites particules (diamètre moyen de l'ordre du micromètre voire inférieur), si possible avec une dispersion de taille resserrée, ce qui se traduit par des surfaces spécifiques élevées : plusieurs dizaines de $\mathrm{m}^{2} \cdot \mathrm{g}^{-1}$ pour un solide non poreux et beaucoup plus dans le cas de solides micro poreux. De nombreux auteurs (Kikic et al., 2000 ; Shekunov et al., 2000b) font également état de la possibilité de co-cristalliser une molécule active et un support (par exemple le lactose). Ceci conduit à un mélange intime qui peut déjà présenter des propriétés supérieures à celles du simple mélange physique des 2 poudres. II est aussi possible de venir imprégner le support par le principe actif, profitant ainsi des propriétés de faible viscosité et de diffusivité massique des FSC.

La seconde utilise des polymères permettant une micro-encapsulation de la molécule active. De plus, des molécules capables de former des complexes par encapsulation moléculaire comme les cyclodextrines sont de plus en plus utilisées. Cette famille de molécules est constituée d'oligomères cycliques de glucose contenant 6,7 ou 8 unités. Ils présentent une cavité tronc conique hydrophobe à l'intérieur, mais hydrophile à l'extérieur. On comprend donc qu'une molécule hydrophobe de taille adaptée pourra venir s'insérer dans la cyclodextrine et présenter ainsi une solubilité apparente grandement augmentée en raison de la présence des groupements hydroxyle hydrophiles de la cyclodextrine. Si la molécule à encapsuler est relativement soluble dans le $\mathrm{CO}_{2}$ supercritique, la complexation peut être réalisée en faisant percoler le mélange supercritique à travers un lit de cyclodextrine, celle-ci étant insoluble dans le $\mathrm{CO}_{2}$. C'est ce qu'ont réalisé Charoenchaitrakool et al. (2002) avec l'ibuprofène. En revanche, une molécule insoluble comme le naproxène devra être co-cristallisée avec la cyclodextrine lors d'un procédé SAS (Foster et al., 2002).

Un autre domaine susceptible d'utiliser ces procédés est la formulation de poudres administrables par voie pulmonaire (Malcolmson et Embleton, 1998). Cependant pour être inhalable, une poudre doit présenter des propriétés difficilement compatibles avec une manipulation aisée (Ebube et al., 1997). En particulier, la densité du produit formulé (principe actif + support ou excipient) doit permettre l'utilisation sous une forme galénique industrialisable, tout en respectant une très faible granulométrie garante de l'absorption par les alvéoles pulmonaires.

D'autres points sont également à prendre en compte. Le problème du changement d'échelle reste posé et rares sont les articles s'intéressant à ce problème. Cependant, Thiering et al. (2001) montrent que l'échelle industrielle peut s'avérer synonyme d'installations de petites tailles, dans la mesure où la production annuelle peut représenter des volumes de l'ordre de la tonne, ce qui est le cas par exemple pour certaines protéines thérapeutiques. Les problèmes réglementaires, notamment de bonnes pratiques de fabrication (BPF ou GMP, good manufacturing practices), sont aussi à considérer, mais ce sont des domaines aujourd'hui bien maîtrisés par les sociétés du secteur. Le coût $d$ 'investissement, souvent présenté comme un frein au développement industriel des FSC, semble de relative faible importance ici, en raison de la valeur ajoutée des molécules traitées qui peut atteindre plusieurs dizaines de milliers d'euros par gramme! De plus, des exemples d'extraction impliquant de gros volumes et des molécules à faible valeur ajoutée ont démontré qu'un développement industriel est possible, même dans ce cas a priori moins favorable.

De nombreuses sociétés pharmaceutiques suivent activement ce domaine de recherches et il est probable que nous soyons proches de la première annonce d'une unité de production industrielle d'un médicament utilisant l'un ou l'autre des procédés décrits dans cet article. Ces dernières années d'ailleurs, deux petites entreprises de haute technologie spécialisées dans ce domaine ont été rachetées par des groupes pharmaceutiques: Bradford Particle Design (BPD, située à Bradford, Royaume-Uni) et Separex (située à Nancy, France), respectivement par Inhale therapeutics et Lavipharm laboratories.

\section{Conclusion}

La génération de particules à partir de FSC est un domaine de recherche né au milieu des années 1980. Après une douzaine d'années consacrées aux premiers défrichages de ce nouvel espace de créativité scientifique, une nouvelle ère semble avoir vu le jour, et plusieurs signes de maturité sont récemment apparus :

- depuis le $4^{\text {th }}$ international symposium on supercritical fluids qui s'est tenu à Sendai (Japon) en 1997, les congrès internationaux consacrés spécifiquement aux FSC réservent une place majeure à la génération de particules, alors qu'auparavant c'était plutôt les applications d'extraction qui prédominaient ;

- la compréhension fine des mécanismes impliqués dans ces procédés, ainsi que leur modélisation, a beaucoup progressé même si la recherche académique dans ces secteurs a encore de beaux jours devant elle ;

- I'intérêt manifesté par l'industrie, et notamment l'industrie pharmaceutique, ne se traduit plus seulement par le parrainage de manifestations scientifiques ou d'équipes de recherche universitaires mais par le rachat de jeunes pousses du domaine.

Cependant, il faut garder à l'esprit que de nombreuses questions, parfois basiques, restent aujourd'hui encore en suspens. Par exemple, I'influence précise des conditions opératoires sur les caractéristiques des particules produites (taille, morphologie, cristallinité, polymorphisme) reste très souvent du domaine de l'empirisme et aucune approche 
globale vraiment satisfaisante n'a encore été proposée à ce jour. L'hydrodynamique des jets de fluide, les mécanismes de nucléation, de croissance ou d'agglomération, les méthodes de récupération des particules ultra-fines et le changement d'échelle de ces procédés doivent encore faire l'objet de nombreux travaux. Ceux-ci, tant au niveau fondamental qu'appliqué, concerneront encore les procédés de type RESS comme ceux de type SAS. Les premiers cités gardent l'avantage de la simplicité de mise en œuvre, alors que la polyvalence des seconds reste leur principal atout. Enfin, n'oublions pas qu'ensemble, ils partagent des avantages considérables: réduction du nombre d'opérations unitaires d'un procédé, pureté des produits obtenus, réduction drastique des éventuels rejets toxiques, possibilité de réaliser des systèmes composés (relargage différé), poudres à propriétés d'usage (solubilité, biodisponibilité) améliorées.

\section{Nomenclature}

$c_{i} \quad$ concentration du soluté, $\left(\mathrm{mol} \cdot \mathrm{m}^{-3}\right)$

$c^{*} \quad$ concentration à la saturation, $\left(\mathrm{mol} \cdot \mathrm{m}^{-3}\right)$

$\Delta_{c} \quad$ sursaturation absolue, $\left(\mathrm{mol} \cdot \mathrm{m}^{-3}\right)$

$E$ facteur d'augmentation

$f_{2} \quad$ fugacité du soluté dans la phase fluide, $(\mathrm{Pa})$

$j$ vitesse de nucléation, $\left(\mathrm{m}^{-3} \cdot \mathrm{s}^{-1}\right)$

$k \quad$ paramètre déterminé expérimentalement, $\left(\mathrm{m}^{3(\mathrm{n}-1)} \cdot \mathrm{mol}^{-\mathrm{n}} \cdot \mathrm{s}^{-1}\right)$

$n \quad$ paramètre déterminé expérimentalement

$P \quad$ pression, $(\mathrm{Pa})$

$P_{2}$ sat $\quad$ pression de sublimation du soluté, $(\mathrm{Pa})$

$R \quad$ constante des gaz parfaits, $\left(\mathrm{J} \cdot \mathrm{mol}^{-1} \cdot \mathrm{K}^{-1}\right)$

$S \quad$ rapport de sursaturation

$T \quad$ température, $(\mathrm{K})$

$V_{0} \quad$ volume du solvant pur avant l'injection de l'anti-solvant, $\left(\mathrm{m}^{3}\right)$

$\checkmark \quad$ volume, $\left(\mathrm{m}^{3}\right)$

$V_{2}{ }^{s} \quad$ volume molaire du solide pur, $\left(\mathrm{m}^{3}\right)$

$y_{2} \quad$ fraction molaire du soluté dans la phase fluide (sans unité)

\section{Symboles grecs :}

$\gamma \quad$ coefficient d'activité

$\mu_{i} \quad$ potentiel chimique du corps i $\left(J \cdot \mathrm{mol}^{-1}\right)$

$\sigma \quad$ sursaturation relative

$\varphi_{2} \quad$ coefficient de fugacité du soluté dans la phase fluide

\section{Références}

Adshiri, T., "Application of Supercritical Fluids in Powder Processing", Kona 16, 89-101 (1998).

Alessi, P., A. Cortesi, I. Kikic, N. Foster, S. Macnaughton et I. Colombo, "Particle Production of Steroid Drugs using Supercritical Fluid Processing", Ind. Eng. Chem. Res. 35, 4718-4726 (1996).

Beach, S., D. Latham, C. Sidgwick, M. Hanna, et P. York, "Control of the Physical Form of Salmeterol Xinafoate", Org. Process Res. Dev. 3, 370-376 (1999).

Beckett, S. T., "The science of Chocolate", Royal Society of Chemistry paperbacks (2000).

Bertucco, A., P. Pallado et L. Benedetti, "Formation of Biocompatible Polymer Microspheres for Controlled Drug Delivery by a Supercritical Antisolvent Technique", dans "High Pressure Chemical Engineering", R. von Rohr et C. Trepp éditeurs, Elsevier, Amsterdam, Netherlands, (1996) pp.217-222.

Bleich, J., P. Kleinebudde et B.W. Muller, "Influence of Gas Density and Pressure on Micro-Particles Produced with the ASES Process ", Int. J. Pharm. 106, 77-86 (1994).

Catchpole, O., S. Hochmann et S. Anderson, "Gas Anti-Solvent Fractionation of Natural Products", dans "High Pressure Chemical Engineering", R. von Rohr et C. Trepp éditeurs, Elsevier, Amsterdam, Netherlands, (1996) pp.309-314.
Chang, C. et A. Randolph, "Precipitation of Microsize Organic Particles from Supercritical Fluids", AIChE J. 35, 1876-1882 (1989).

Chang, C. et A. Randolph, "Solvent Expansion and Solute Solubility Predictions in Gas-Expanded Liquids", AIChE J. 36, 939-942 (1990).

Charoenchaitrakool, M., F. Dehghani, N.R. Foster et H.K. Chan, "Micronization by Rapid Expansion of Supercritical Solutions to Enhance the Dissolution Rates of Poorly Water Soluble Phamaceuticals", Ind. Eng. Chem. Res. 39, 4794-4802 (2000).

Charoenchaitrakool, M., F. Dehghani et N.R. Foster, "Utilization of Supercritical Carbon Dioxide for Complex Formation of Ibuprofen and Methyl-b-Cyclodextrin", Int. J. Pharma. 239, 103-112 (2002).

Chhor, K., J.F. Bocquet, et C. Pommier, "Syntheses of Submicron Titania Powders in Vapor, Liquid and Supercritical Phases, a Comparative Study", Mat. Chem. Phys. 32, 249-254 (1992).

Chhor, K., J.F. Bocquet, et C. Pommier, "A New TiO2 Film Deposition Process in a Supercritical Fluid", Surface Coatings Technol. 70, 73-78 (1994).

Chhor, K., J.F. Bocquet et C. Pommier, "Syntheses of Submicron Magnesium Oxide Powders", Mat. Chem. Phys. 40, 63-68 (1995).

Chrastil, J.,"Solubility of Solids and Liquids in Supercritical Gases", J. Phys. Chem. 86, 3016-3021 (1982).

Cygnarowicz, M. "Equilibrium Solubilities of b-Carotene in Supercritical Carbon Dioxide", Fluid Phase Equilib. 59, 57-71(1990).

Debenedetti, P. "Homogeneous Nucleation in Supercritical Fluids", AlChE J. 35, 1289-1298 (1990).

Debenedetti, P., J. Tom, X. Kwauk et S.D. Yeo, "Rapid Expansion of Supercritical Solutions (RESS) : Fundamentals and Applications", Fluid Phase Equilib. 82, 311-321 (1993a).

Debenedetti, P., J. Tom, S.D. Yeo et G.B. Lim, "Application of Supercritical Fluids for the Production of Sustained Delivery Devices", J. Controlled Release 24, 27-44 (1993b).

De La Fuente Badilla, J.C., C.J. Peters et J. De Swaan Arons, "Volume Expansion in Relation to the Gas-Antisolvent Process ", J. Supercrit. Fluids 17, 13-23 (2000).

Dixon, D. et K. Johnston, "Molecular Thermodynamics of Solubilities in Gas Antisolvent Cristallisation", AIChE J. 37, 1441-1449 (1991).

Dixon, D., K. Johnston et R. Bodmeier, "Polymeric Materials Formed by Precipitation with a Compressed Fluid Antisolvent", AIChE J. 39, 127-139 (1993).

Domingo, C., E. Berends et G.M. Van Rosmalen, "Precipitation of Ultra-Fine Organic Crystals from the Rapid Expansion of Supercritical Solutions Over a Capillary and a Frit Nozzle", J. Supercrit. Fluids 10, 39-55 (1997).

Ebube, N., A. Hikal, C. Wyandt, D. Beer, L. Miller et A. Jones, "Effect of Drug, Formulation and Process Variables on Granulation and Compaction Characteristics of Heterogeneous Matrices. Part 1: HPMC and HPC System", Int. J. Pharm. 156, 49-57 (1997).

Ekart, M., K. Bennett, S. Ekart, G. Gurdial, C. Liotta, et C. Eckert, "Cosolvent Interactions in Supercritical Fluid Solutions", AIChE J. 39, 235-248 (1993).

Fages, J., A. Marty, C. Delga, J.S. Condoret, D. Combes et P. Frayssinet, "Use of Supercritical CO2 for Bone Delipidation", Biomaterials 15, 650-656 (1994).

Fages, J., E. Jean, P. Frayssinet, D. Mathon, B. Poirier, A. Autefage et D. Larzul, "Bone Allografts and Supercritical Processing : Effects on Osteointegration and Viral Safety", J. Supercrit. Fluids 13, 351-356 (1998a).

Fages, J., B. Poirier, Y. Barbier, P. Frayssinet, M.L. Joffret, W. Majewski, G. Bonel et D. Larzul, "Viral Inactivation of Human Bone Tissue using Supercritical Fluid Extraction", ASAIO J. 44, 289-293 (1998b).

Foster, N.R., R. Mammucari et F. Deghani, "Coprecipitaion of Pharmaceuticals using Gas Antisolvent Technique", dans "Proc. $8^{\text {th }}$ meeting on Supercritical Fluids", M. Besnard et F. Cansell, Bordeaux, France, 14-17 avr. ISASF, (2002) pp.321-326.

Gallagher, P.M., M.P. Coffey, V.J. Krukonis et W.W. Hillstrom, "GasAntisolvent Recristallisation of RDX : Formation of Ultra-Fine Particles of a Difficult-to-Comminute Explosive", J. Supercrit. Fluids 5, 130-136 (1992). 
Guan, B., B. Han et H. Yan, "Effect of Acetic Acid+Acetonitrile and Ethanol + Acetonitrile Mixed Cosolvents on the Solubility of Stearic Acid in Supercritical CO ${ }^{\prime \prime}$, Fluid Phase Equilib. 149, 277-286 (1998).

Hanna, M., P. York et B. Shekunov, "Control of the Polymorphic Forms of a Drug Substance by Solution Enhanced Dispersion by Supercritical Fluids (SEDS)", dans Proc. 5th meeting on Supercritical Fluids", M. Perrut et P. Subra, Nice, France, 23-25 mars, ISASF, (1998) pp. 325-330.

Hannay, J. B. et Hogarth, J. "On the Solubility of Solids in Gases", Proc. R. Soc. London 29, 324-326 (1879).

Helfgen, B., P. Hils, C. Holzknecht, M. Türk et K. Schaber, "Simulation of Particle Formation during the Rapid Expansion of Supercritical Solutions", Aerosol Sci. 32, 295-319 (2001).

Jung, J. et M. Perrut, "Particle Design using Supercritical Fluids : Literature and Patent Survey", J. Supercrit. Fluids 20, 179-219 (2001).

Kikic, I., M. Moneghini, B. Perissuti et D. Voinovich, "Improvement of Carmabazepine Dissolution Characteristics using Supercritical Carbon Dioxide", dans "Proc. 7th meeting on Supercritical Fluids", M. Perrut et E. Reverchon, Antibes, France, 6-8 déc., ISASF, (2000) pp.95-100.

Kim, J.H., T.E. Paxton et D.L. Tomasko, "Microencapsulation of Naproxen using Rapid Expansion of Supercritical Solutions", Biotechnol. Prog. 12, 650-661 (1996).

Klein, J. P. et R. David, "Reaction Crystallization", dans "Crystallization Technology Handbook", A. Mersmann, éditeur,. Marcel Dekker Inc., New York, NY, (1995) pp. 359-400.

Knez, Z. "Micronisation of Pharmaceuticals using Supercritical Fluids", dans "Proc. 7th meeting on Supercritical Fluids", M. Perrut et E. Reverchon, Antibes, France, 6-8 déc., ISASF, (2000) pp.21-26.

Knutson, B., P. Debenedetti et J. Tom, "Preparation of Microparticulates Using Supercritical Fluids", dans "Microparticulate Systems for the Delivery of Proteins and Vaccines, Drugs and the Pharmaceutical Sciences Series", S. Cohen et H. Bernstein éditeurs, Marcel Dekker Inc., New York, Ny, (1996), 77, pp.89-125.

Kokot, K., Z. Knez et D. Bauman, "S-L-G (Solid-Liquid-Gas) Phase Transition of Cocoa Butter in Supercritical CO2", Acta Alimentaria 28, 197-208 (1999).

Kordikowski, A., A.P. Schenk, R. M. Van Nielen et C.J. Peters, "Volume Expansions and Vapor-Liquid Equilibria of Binary Mixtures of a Variety of Polar Solvents and Certain Near-Critical Solvent", J. Supercrit. Fluids 8, 205-216 (1995).

Kordikowski, A., B. Shekunov et P. York, "Cristallisation of Sulfathiazole Polymorphs using $\mathrm{CO} 2$ ", dans "Proc. $7^{\text {th }}$ meeting on Supercritical Fluids", M. Perrut et E. Reverchon, Antibes, France, 6-8 déc., ISASF, (2000) pp.117-122.

Kroeber, H., U. Teipel et H. Krause, "Formation of Submicron Particles Obtained by the Rapid Expansion of Supercritical Solutions", Proceedings of the 14th symposium on Industrial Crystallization : IChemE Cambridge, UK (1999).

Krukonis, V. "Supercritical Fluid Nucleation of Difficult-to-Comminute Solids", AIChE annual meeting San Francisco, (1984).

Ksibi, H, C. Tenaud, P. Subra et Y. Garrabos, "Numerical Simulation of the Rapid Expansion of Supercritical Fluid Flow", Eur. J. Mechanics/ B Fluids 15, 569-596 (1996).

Kwauk, X. et P. Debenedetti, "Mathematical Modeling of Aerosol Formation by Rapid Expansion of Supercritical Solutions in a Converging Nozzle", J. Aerosol. Sci. 24, 445-469 (1993).

Lele, A.K. et A.D. Shine, "Morphology of Polymers Precipitated from a Supercritical Solvent", AIChE J. 38, 742-752 (1992).

Lele, A.K. et A.D. Shine, "Effect of RESS Dynamics on Polymer Morphology", Ind. Eng. Chem. Res. 33,1476-1485 (1994).

Loisel, C., G. Keller, G. Lecq, C. Bourgaux et M. Ollivon, "Phase Transitions and Polymorphism of Cocoa Butter", JAOCS 75, 425-439 (1998).

Lora, M., A. Bertucco et I. Kikic, "Simulation of the Semi-Continuous Supercritical Antisolvent Recrystallization Process", Ind. Eng. Chem. Res. 39, 1487-1496 (2000).

Loth, H. et E. Hemgesberg, "Properties and Dissolution of Drugs Micronized by Cristallisation from Supercritical Gases", Int. J. Pharm. 32, 265-270 (1986).
Lucien, F. P. et N.R. Foster, "Solubility of Solid Mixtures in Supercritical Carbon Dioxide : a Review", J. Supercrit. Fluids 17, 111-134 (2000). McHugh, M. A. et V. J. Krukonis, "Supercritical Fluid Extraction : Principles and Practice", $2^{\text {nd }}$ edition, Butterworth-Heinemann, Stoneham, MA, (1994).

Magnan, C., E. Badens et G. Charbit, "Soy Lecithin Precipitation with a Compressed Fluid Anti-Solvent. Influence of Process Parameters", J. Supercrit. Fluids 19, 69-78 (2000).

Malcolmson, R. et J. Embleton, "Dry Powder Formulations for Pulmonary Delivery", PSTT 9, 394-398 (1998).

Matson, D., J. Fulton, R. Petersen et R. Smith, "Rapid Expansion of Supercritical Fluid Solutions : Solute Formation of Powders, Thin Films and Fibers", Ind. Eng. Chem. Res. 26, 2298-2306 (1987a).

Matson, D., R. Petersen R. et R.Smith, "Production of Powders and Films by the Rapid Expansion of Supercritical Solutions", J. Mat. Sci. 22, 1919-1928 (1987b).

Mendes, R., B. Nobre, J. Coelho et A. Palavra, "Solubility of b-Carotene in Supercritical Carbon Dioxide and Ethane", J. Supercrit. Fluids 16, 99-106 (1999).

Méndez-Santiago, J. et A. Teja, "Solubility of Solids in Supercritical Fluids : Consistency of Data and a New Model For Cosolvent Systems", Ind. Eng. Chem. Res. 39, 4767-4771 (2000).

Mishima, K., K. Matsuyama, D. Tanabe, S. Yamauchi, T.J. Young et K.P. Johnston, "Microencapsulation of Proteins by Rapid Expansion of Supercritical Solution with a Non-Solvent", AIChE J. 46, 857-865 (2000).

Mohamed, R., P. Debenedetti et R. Prud'homme, "Effects of Process Conditions on Crystals Obtained from Supercritical Mixtures", AIChE J. 35, 325-328 (1989).

Morris, K., S. Nail, G. Peck, S. Byrn, U. Griesser, J. Stowell, S.J. Hwang et K. Park, "Advances in Pharmaceutical Materials and Processing", PSTT 6, 235-245 (1998).

Neau, E., S. Garnier et L. Avaullée, "A Consistent Estimation of Sublimation Pressures using a Cubic Equation of State and Fusion Properties", Fluid Phase Equilib. 164, 173-186 (1999).

Palakodaty, S., J. Pritchard, P. York et M. Hanna, "Cristallisation of Lactose Using Solution Enhanced Dispersion by Supercritical Fluids (SEDS) Technique", dans "Proc. 5th meeting on Supercritical Fluids", M. Perrut et P. Subra, Nice, France, 23-25 mars, ISASF, (1998a) pp. 275-280.

Palakodaty, S., P. York et J. Pritchard, "Supercritical Fluid Processing of Materials from Aqueous Solutions: The Application of SEDS to Lactose as a Model Substance", Pharm. Res. 15, 1835-1843 (1998b).

Papet, S., A. Julbe, S. Sarrade et C. Guizard, "Synthesis of Submicronic Titanium Hydroxide and Oxide Powders in SC CO2", dans "Proc. $7^{\text {th }}$ meeting on Supercritical Fluids" M. Perrut et E. Reverchon, Antibes, France, 6-8 déc., ISASF, (2000) pp.173-178.

Peng, D.Y. et D. Robinson. "A New Two-Constant Equation of State" Ind. Eng. Chem., Fundam. 15, 59-64 (1976).

Raynie, D, "Warning Concerning the Use of Nitrous Oxide in Supercritical Fluid Extractions", Analytical Chemistry 65, 3127-3128 (1993).

Reverchon, E., G. Della Porta, R. Taddeo, P. Pallado et A. Stassi, " Solubility and Micronisation of Griseofulvin in Supercritical CHF3", Ind. Eng. Chem. Res. 34, 4087-4091 (1995).

Reverchon, E., G. Della Porta et M.G. Falivene, "Process Parameters Controlling the Supercritical Antisolvent Micronisation of Some Antibiotics", dans "Proc. 6th Meeting on Supercritical Fluids", M Poliakoff, M.W. George et S.M. Howdle, Nottingham, UK, (1999), pp.157-162.

Reverchon E., "Supercritical Antisolvent Precipitation of Micro- and Nano-Particles", J. Supercrit. Fluids 15, 1-21 (1999).

Reverchon, E., "Supercritical Assisted Atomisation to Produce Microand/or Nano-Particles of Controlled Size and Distribution", Ind. Eng. Chem. Res. 41, 2405-2411 (2002)

Reverchon, E. et M. Perrut, "Particle Design using Supercritical Fluids : Review and Examples", dans "Proc. $7^{\text {th }}$ meeting on Supercritical Fluids", M. Perrut et E. Reverchon, Antibes, France, 6-8 déc., ISASF, (2000b) pp.3-20. 
Richard, J., B. Pech, C. Thies, M.I. Ribeiro Dos Santos et J.P. Benoit, "Preparation and Characterisation of Sustained-Release Microcapsules Obtained using Supercritical Fluid Technology", dans "Proc. $7^{\text {th }}$ meeting on Supercritical Fluids", M. Perrut et E. Reverchon, Antibes, France, 6-8 déc., ISASF, (2000b) pp.143-146.

Ruchatz, F., P. Kleinebudde et B. Müller, "Residual Solvents in Biodegradable Microparticles. Influence of Process Parameters on the Residual Solvent in Microparticles Produced by the Aerosol Solvent Extraction System (ASES) Process", J. Pharm. Sci. 86, 101-105 (1997).

Sauceau, M., J. Fages, J. J. Letourneau et D. Richon, "A Novel Apparatus for Accurate Measurements of Solid Solubilities in Supercritical Phases", Ind. Eng. Chem. Res. 39, 4609-4614 (2000a).

Sauceau, M., J. J. Letourneau, D. Richon et J. Fages, "Solubility of a Pharmaceutical Solid in Supercritical Mixtures : Measurements and Modelling", dans "Proc. $7^{\text {th }}$ meeting on Supercritical Fluids", M. Perrut et E. Reverchon, Antibes, France, 6--8 déc., ISASF, (2000b) pp. 959-964.

Sauceau, M., J. J. Letourneau, D. Richon et J. Fages, "Effects of Two Cosolvents on the Solubility Behaviour of an Active Substance in Supercritical $\mathrm{CO} 2$ ", dans $7^{\text {th }}$ Conference of Food Engineering, AIChE Annual Meeting, Reno (USA), 4-9 November (2001) pp 212-217.

Shekunov, B., Y. Sun, E. Astracharchik, P. York et J. Baldyga, "Optical Characterisation and Mechanism of Antisolvent Precipitation in Turbulent Flow", dans "Proc. $7^{\text {th }}$ meeting on Supercritical Fluids", M. Perrut et E. Reverchon, Antibes, France, 6-8 déc., ISASF, (2000a) pp.65-70.

Shekunov, B., S. Bristow, P. York et A.H.L. Chow, "Precipitation of Acetaminophen and p-Acetoxyacetanilide using Supercritical $\mathrm{CO}_{2}$ ", dans "Proc. 7th meeting on Supercritical Fluids", M. Perrut et E. Reverchon, Antibes, France, 6-8 déc., ISASF, (2000b) pp.111-116.

Shekunov, B., J. Baldyga et P. York, "Particle Formation by Mixing with Supercritical Antisolvent at High Reynolds Numbers", Chem. Eng. Sci. 56, 2421-2433 (2001).

Sievers, R.E., P.D. Milewski, S.P. Sellers, B.A. Miules, B.J. Korte, K.D. Kusek, G.S. Clack, B. Mioskowski et J.A. Villa, "Supercritical and Near-Critical Carbon Dioxide Assisted Low-Temperature Bubble Drying", Ind. Eng. Chem. Res. 39, 4831-4836 (2000).

Skerget, M., Z. Knez et M. Habulin, "Solubility of b-Carotene and Oleic Acid in Dense CO2 and Data Correlation by a Density Based Model", Fluid Phase Equilib. 109, 131-138 (1995).

Sloan, R., M. Hollowood, G. Humpreys, W. Ashraf et P. York, "Supercritical Fluid Processing : Preparation of Stable Protein Particles", dans "Proc. $5^{\text {th }}$ meeting on Supercritical Fluids", M. Perrut et P. Subra, Nice, France, 23-25 mars, ISASF, (1998) pp. 301-306.

Sloan, R., M. Tservistas, M. Hollowood, L. Sarup, G.O. Humpreys, P. York, W. Ashraf et M. Hoare, "Controlled Particle Formation of Biological Material using Supercritical Fluids", dans "Proc. $6^{\text {th }}$ Meeting on Supercritical Fluids", M Poliakoff, M.W. George et S.M. Howdle, Nottingham, UK, (1999), pp.169-180.

Smith, R., J. Fulton, R. Petersen, A. Kopriva et B. Wright, "Performance of Capillary Restrictors in Supercritical Fluid Chromatography", Anal. Chem. 58, 2057-2064 (1986).

Subra, P. et P. Debenedetti, "Application of RESS to Several Low Molecular Weight Compounds", dans "High Pressure Chemical Engineering", R. von Rohr et C. Trepp éditeurs, Elsevier, Netherlands, Amsterdam, (1996) pp.49-54.

Subra, P. et P. Jestin, "Powders Elaboration in Supercritical Media : Comparison with Conventional Routes", Powder Technol. 103, 2-9 (1999).
Sun, Y.P., R. Guduru, F. Lin et T. Whiteside, "Preparation of Nanoscale Semiconductors through the Rapid Expansion of Supercritical Expansion (RESS) into Liquid Solution", Ind. Eng. Chem. Res. 39, 4663-4669 (2000).

Sze, Tu L., F. Dehgani, A.K. Dillow et N.R. Foster, "Applications of Dense Gases in Pharmaceutical Processing.", dans "Proc. $5^{\text {th }}$ meeting on Supercritical Fluids", M. Perrut et P. Subra, Nice, France, 23-25 mars, ISASF, (1998) pp. 263-269.

Thiering, R., M. Charoenchaitrakool, L. Sze Tu, F. Deghani, A.K. Dillow et N.R. Foster, "Crystallization of p-Hydroxybenzoic Acid by Solvent Expansion with Dense Carbon Dioxide", dans "Proc. $5^{\text {th }}$ meeting on Supercritical Fluids", M. Perrut et P. Subra, Nice, France, 23-25 mars, ISASF, (1998) pp. 291-296

Thiering, R., F. Deghani et N.R. Foster, "Current Issues Relating to Anti-Solvent Micronisation Techniques and their Extension to Industrial Scales", J. Supercrit. Fluids 21, 159-177 (2001).

Ting, S., S. Macnaughton, D. Tomasko et N.R. Foster, "Solubility of Naproxen in Supercritical Carbon with and without Cosolvents", Ind. Eng. Chem. Res. 32, 1471-1481 (1993).

Tom, J.W. et P. G. Debenedetti, "Particle Formation with Supercritical Fluids - a review", J. Aerosol. Sci. 22, 555-584 (1991).

Tom, J., G. Lim, P. Debenedetti et R.K. Prud'homme, "Applications of Supercritical Fluids in Controlled Release of drugs", dans "Supercritical Fluid Engineering Science", ACS Symp. Ser. 214. Washington DC (1992).

Tsutsumi, A., S. Nakamoto, T. Mineo et K. Yoshida, "A Novel FluidizedBed Coating of Fine Particles by Rapid Expansion of Supercritical Fluid Solutions", Powder Technol. 85, 275-278 (1995).

Van Alsten, J. et C. Eckert, "Effect of Entrainers and of Solute Size and Polarity in Supercritical Fluid Solutions", J. Chem. Eng. Data 38, 605-610 (1993).

Van Den Bos, V., M. Prandi et L. N'Kaoua, "Le CO2 supercritique entre dans I'atelier de peinture", L'usine nouvelle 2333, 46-47 (1996).

Warwick, B., F. Dehghani, N.R. Foster, J.R. Biffin et H.L. Regtop, "Synthesis, Purification and Micronization of Pharmaceuticals using the Gas Antisolvent Technique", Ind. Eng. Chem. Res. 39, 4571-4579 (2000).

Weber, A., C. Nolte, M. Bork et R. Kümmel, "Recovery of Lecithin from Egg-Yolk Extracts by Gas Antisolvent Cristallisation", dans "Proc. 6 th $^{\text {th }}$ Meeting on Supercritical Fluids", M Poliakoff, M.W. George et S.M. Howdle, Nottingham, UK, (1999), pp.181-184.

Weidner, E., R. Steiner et Z. Knez, "Powder Generation from Polyethyleneglycols with Compressible Fluids", dans "High Pressure Chemical Engineering", R. von Rohr et C. Trepp éditeurs, Elsevier, Netherlands, Amsterdam (1996) pp.223-228.

Werling, J.O. et P.G. Debenedetti, "Numerical Modeling of Mass Transfer in the Supercritical Antisolvent Process", J. Supercrit. Fluids 16, 167-181 (1999).

Werling, J.O. et P.G. Debenedetti, "Numerical Modeling of Mass Transfer in the Supercritical Antisolvent Process: miscible conditions", J. Supercrit. Fluids 18, 11-24 (2000).

Winters, M.A., B.L. Knutson, P. Debenedetti, H.G. Sparks et T.M. Przybycien, "Precipitation of Proteins in Supercritical Carbon Dioxide", J. Pharm. Sci. 85, 586-595 (1996).

Yeo, S.D., G.B. Lim, P. Debenedetti et H. Bernstein, "Formation of Microparticulate Protein Powders Using a Supercritical Fluid Antisolvent", Biotech. Bioeng. 41, 341-346 (1993).

Manuscript received September 16, 2002; revised manuscript received February 25, 2002; accepted for publication February, 2003. 